\title{
Stathmin recruits tubulin to Listeria monocytogenes-induced actin comets and promotes bacterial dissemination
}

Ana Catarina Costa $^{1}$, Filipe Carvalho ${ }^{1, \#}$, Didier Cabanes ${ }^{1,}$, Sandra Sousa ${ }^{1, *}$

${ }^{1}$ Group of Molecular Microbiology, i3S - Instituto de Investigação e Inovação em Saúde, IBMC - Instituto de Biologia Molecular e Celular, Universidade do Porto, Rua Alfredo Allen, 208, 4200-135 Porto, Portugal.

\# Current address : Unité des Interactions Bactéries-Cellules, Institut Pasteur, 25 Rue du Dr Roux 75015

Paris, France

* Corresponding author: srsousa@ibmc.up.pt

Running title: Stathmin recruits tubulin to $\mathrm{Lm}$ comets

ORCID:

Ana Costa 0000-0003-0062-8714

Filipe Carvalho 0000-0002-6828-1772

Didier Cabanes 0000-0002-4001-1332

Sandra Sousa 0000-0001-8578-0461

\author{
Abbreviations: \\ Lm-Listeria monocytogenes \\ MT - microtubule \\ $\mathrm{ABM}$ - actin-based motility \\ MOI - multiplicity of infection
}




\begin{abstract}
The tubulin cytoskeleton is one of the main components of the cytoarchitecture and is involved in several cellular functions. Here we examine the interplay between Listeria monocytogenes $(\mathrm{Lm})$ and the tubulin cytoskeleton upon cellular infection. We show that non-polymeric tubulin is present throughout $\mathrm{Lm}$ actin comet tails and, to a less extent, in actin clouds. Moreover, we demonstrate that stathmin, a regulator of microtubule dynamics, is also found in these $\mathrm{Lm}$-associated actin structures and is required for tubulin recruitment. Depletion of host stathmin results in longer comets containing less F-actin, which may be correlated with higher levels of inactive cofilin in the comet, thus suggesting a defect on local F-actin dynamics. In addition, intracellular bacterial speed is significantly reduced in stathmin-depleted cells, revealing the importance of stathmin/tubulin in intracellular $\mathrm{Lm}$ motility. In agreement, the area of infection foci and the total bacterial loads are also significantly reduced in stathmin-depleted cells. Collectively, our results demonstrate that stathmin promotes efficient cellular infection, possibly through tubulin recruitment and control of actin dynamics at Lmpolymerized actin structures.
\end{abstract}

\title{
Keywords
}

Listeria monocytogenes, tubulin, stathmin, cytoskeleton, actin comet tails, infection 


\section{INTRODUCTION}

During cellular infection, pathogenic bacteria, virus and parasites recurrently exploit the host cytoskeleton composed by microfilaments (actin), microtubules (MTs; tubulin), intermediate filaments and septins, which together provide structural support and control numerous cellular processes [1]. To target host cytoskeleton, pathogens deploy specialized effector proteins to differentially hijack these filaments or their regulators at different steps of the infectious cycle [2-5]. As the different components of the host cytoarchitecture establish regulatory and structural cross-talks, cytoskeleton hijacking by pathogens is frequently accompanied by cooperation between filaments and interplay of common signaling pathways [1,6]. Ultimately, pathogen modulation of cytoskeletal dynamics during the course of infection promotes adherence to host cells, internalization, autophagy escape, intracellular spreading and tissue dissemination [7].

Listeria monocytogenes $(\mathrm{Lm})$ is a human bacterial pathogen capable of invading, replicating and disseminating in a variety of cell types, ultimately causing serious illness [8]. These properties are related to the ability of $\mathrm{Lm}$ to usurp the host actin cytoskeleton [8]. Indeed, through the expression of the surface protein ActA, Lm activates the host cytosolic Arp2/3 complex to nucleate actin [9]. Filamentous actin (F-actin) assembles initially in the form of a cloud surrounding the bacterium and, subsequently, at a single pole forming a comet-like tail [10]. Such polarized actin polymerization generates the propulsion force necessary for the bacterium to move and spread into adjacent cells, thus enabling the dissemination of infection [11].

Simplified systems using purified proteins have elucidated that, in addition to Arp2/3 complex, cofilin and capping protein are essential for $L m$ actin-based motility (ABM), while VASP and profilin are important to regulate ABM [12]. Cofilin acts as a F-actin-depolymerizing factor and capping protein avoids unnecessary growth of branched filaments, whereas VASP and profilin catalyze the addition of actin monomers to growing filaments [11]. However, in the context of cellular infections, other host proteins, such as lamellipodin and formins mDia1-3, were shown to integrate the $\mathrm{Lm}$ comet tail and to assist an effective bacterial propulsion and dissemination $[13,14]$.

The involvement of the tubulin cytoskeleton and its regulators in $L m$ infection has never been deeply addressed, however several lines of evidence point to their possible interconnection. Indeed, descriptive studies have shown that the MT-depolymerizing drug, nocodazole, inhibits $\mathrm{Lm}$ internalization in endothelial cells, macrophages and dendritic cells [15-17], and significantly decreases intracellular speed [18]. Moreover, despite Lm movement does not require MTs [19], proteomic analysis of isolated $L m$ comet tails revealed an unexpected high abundance of tubulin $(\sim 5 \%)$, as well as the presence of the MT-interacting proteins Tau and dynamin [20]. Finally, stathmin that regulates MT dynamics by sequestering free tubulin heterodimers promoting MT disassembly [21,22], was predicted to interact with LaXp180, which in turn interact with ActA [23,24].

Herein, we demonstrate and characterize for the first time the presence of tubulin in $\mathrm{Lm}$ actin clouds and comet tails. We also identify stathmin as a novel component of the comet tail responsible for the downstream recruitment of tubulin. Importantly, we show that stathmin modulates actin dynamics at the comet tail, ultimately impacting $\mathrm{Lm}$ dissemination in an epithelial cell monolayer. 


\section{EXPERIMENTAL PROCEDURES}

\section{Bacterial strains, cell lines and growth conditions}

Listeria monocytogenes ( $\mathrm{m}$ ) EGDe [25], EGDe-GFP (constitutively expressing GFP) [26], EGDe $\triangle a c t$ A [26], EGD [27] and 10403S [28] were grown in Brain Heart Infusion (BHI, Difco Laboratories) media at $37^{\circ} \mathrm{C}$ under aerobic conditions with shaking. Human colorectal adenocarcinoma cell line Caco-2 (ATCC HTB-37) was propagated in Eagle's Minimum Essential Medium (EMEM) supplemented with 20\% (v/v) fetal bovine serum (FBS), $1 \mathrm{mM}$ sodium pyruvate and $0.1 \mathrm{mM}$ non-essential amino acids. Human choriocarcinoma cell line Jeg-3 (ATCC HTB-36) was cultured in EMEM supplemented with 10\% FBS. Mouse macrophage J774, rat kangaroo Ptk2 and HeLa cell lines were cultured in Dulbecco's Modified Eagle's Medium (DMEM) supplemented with $10 \%$ FBS. Human choriocarcinoma BeWo cells (ATCC CCL-98) were grown in DMEM/HamF12 (50/50) supplemented with $10 \% \mathrm{FBS}$ and $2 \mathrm{mM}$ glutamine. Cells were kept at $37^{\circ} \mathrm{C}$ in a $5 \% \mathrm{CO}_{2}$ humidified atmosphere. Cell culture media and supplements were purchased from Lonza. Whenever stated, cells were treated with $33 \mu \mathrm{M}$ nocodazole (Sigma) or $0.27 \mu \mathrm{M}$ colcemid KaryoMAX (Gibco) for $2 \mathrm{~h}$.

\section{Infection of cells}

Cells $\left(4 \times 10^{5}\right)$ were seeded in 6-well plates and propagated for $24 \mathrm{~h}$. $L m$ was grown to an optical density at 600 $\mathrm{nm}$ (OD600) of 0.7, washed and diluted in FBS-free media. Cells were washed three times with FBS-free media and incubated with $L m$ suspension for $1 \mathrm{~h}$ at different multiplicities of infection (MOIs): 50 (HeLa, BeWo, Ptk2), 30 (Caco-2), 10 (J774) and 2 (Jeg-3). Bacteria-containing media were replaced by complete media containing $50 \mu \mathrm{g} / \mathrm{mL}$ gentamicin and infection was allowed to proceed until 2 or $7 \mathrm{~h}$. For $24 \mathrm{~h}$ infections, MOIs of 0.5-5 were used.

\section{SiRNA transfection}

HeLa cells were seeded in 6 -well plates $\left(3 \times 10^{5}\right.$ cells/well) or in 4 -well $\mu$-slides (Ibidi, $4 \times 10^{4}$ cells/well). Transfection was performed $30 \mathrm{~min}$ later using HiPerfect (Qiagen), according to the manufacturer's protocol. Stathmin (sc-36127) and control (sc-44232) siRNAs were purchased from Santa Cruz Biotechnology and used at a final concentration of $50 \mathrm{nM}$. Stathmin siRNA comprises a pool of 3 oligonucleotide pairs: 5'CCAGAUCCAGACUGUAAGAtt-3', 5'-GGGAGAAACUGAaAGUGUUtt-3' and 5'CCUCCUGGUUGAUACUUGUtt-3'. Cells were infected with $L m 48 \mathrm{~h}$ after transfection.

\section{Cold stability assay and immunofluorescence}

For cold stability assay, infected cells were incubated for $10 \mathrm{~min}$ in complete medium at $4^{\circ} \mathrm{C}$, on top of ice, and fixed with 3\% (v/v) paraformaldehyde (PFA) at room temperature. Alternatively, cells were immediately fixed with $3 \% \mathrm{PFA}$ at $37^{\circ} \mathrm{C}$ to keep microtubule integrity. Fixed coverslips were quenched with $50 \mathrm{mM} \mathrm{NH}_{4} \mathrm{Cl}(20$ $\mathrm{min}$ ), permeabilized with $0.3 \%(\mathrm{v} / \mathrm{v})$ Triton X-100 (5 min), washed in phosphate buffered saline (PBS) and incubated with blocking solution (1\% BSA and 20\% FBS in PBS) for 30 min. Coverslips were incubated with 
primary antibodies raised against stathmin (ab52906), Lm (ab35132), phospho-S3 cofilin (ab12866) (Abcam), tyrosinated tubulin (MCA77G, BioRad), $\alpha$-tubulin (T5168), acetylated tubulin (T7451) or detyrosinated tubulin (AB3201) (Sigma Aldrich), washed and incubated with AlexaFluor 488- (Invitrogen), Cy3- and/or Cy5(Jackson ImmunoResearch) conjugated secondary antibodies, AlexaFluor 488- (Invitrogen) or TRITCphalloidin (Thermo Fischer) and $2 \mathrm{ng} / \mathrm{mL}$ DAPI (Sigma Aldrich). Coverslips for STED microscopy were incubated with anti-mouse STAR 580 (2-0002-005-1), anti-rabbit STAR 635P (2-0012-007-2) (Abberior), SiRactin (SC001, Cytoskeleton) and WGA-FITC (L4895, Sigma Aldrich). Coverslips were mounted with AquaPoly/Mount medium (18606, Polysciences).

\section{Microscopy and image analyses}

Epi-fluorescence microscopy was performed using Zeiss AxioImager Z1 microscope equipped with a 63x (1.4NA) objective. Z-stack images were acquired and deconvoluted using Huygens software to decrease blurring and noise. Olympus BX53 microscope coupled with a 20x (0.17NA) lens was used to obtain full coverslip imaging by multiple image alignment. Super-resolution stimulated emission depletion (STED) microscopy was performed using an objective 63x (1.4NA) of Abberior Instruments. Fiji software [29] was used for single and Z-stack image manipulation and analyses by drawing specific regions of interest (ROI) and calculating their mean fluorescence intensity (MFI) and RGB profiles. For live cell imaging, $0.4 \times 10^{5}$ cells were seeded in $\mu$-slides (Ibidi) and observed under a Leica DMI 6000B motorized microscope coupled to an environmental control chamber maintaining cells at $37^{\circ} \mathrm{C}$ in a $5 \% \mathrm{CO}_{2}$ humidified atmosphere. Phase-contrast images were acquired every $20 \mathrm{sec}$, at multiple points for $7 \mathrm{~h}$. Fiji software was used to compile time-lapse images and analyze the resulting movies with the manual tracking plugin.

\section{Immunoblot analysis}

Cells were directly lysed in Laemmli buffer [3\% (v/v) glycerol, 5\% (v/v) mercaptoethanol, 2\% (v/v) SDS, 0.1\% (w/v) bromophenol blue in $100 \mathrm{mM}$ Tris-HCl, $\mathrm{pH}$ 6.8]. Protein samples were boiled, resolved by SDS-PAGE

(10\% polyacrylamide gel) and transferred onto nitrocellulose membrane (Bio-Rad Laboratories). Membranes were blocked in 5\% skimmed milk in TBS-T (20 mM Tris-HCl, pH 7.4, $137 \mathrm{mM} \mathrm{NaCl,} \mathrm{0.1 \%} \mathrm{Triton} \mathrm{X-100),}$ immunoblotted with primary antibodies raised against stathmin (ab52906, Abcam) and GAPDH (sc-32233, Santa Cruz Biotechnology) and horseradish peroxidase-conjugated secondary antibodies (BI2413C and BI2407, PARIS Biotech). Chemiluminescence (Pierce ECL Western Blotting Substrate, Thermo Fischer Scientific) was used for protein detection, and band intensities were quantified using Image J software [30].

\section{Spreading and intracellular viability assays}

Intercellular spreading assay was performed as reported [31]. Briefly, confluent HeLa cells were infected under low MOI (0.5) for $24 \mathrm{~h}$, fixed with 3\% PFA and labeled with anti- Lm antibodies (ab35132, Abcam) for immunofluorescence analysis. Regions of dense $\mathrm{Lm}$ labeling were defined as foci of infection and their area was calculated using Image $\mathbf{J}$ software [30]. Intracellular bacteria were quantified by enumeration of viable bacteria after cell lysis in $0.2 \%$ Triton X-100 and plating on BHI agar as described previously [32]. 


\section{Statistical analyses}

Statistical analyses were performed with Prism 7 software (GraphPad software, Inc.). Two-tailed Student's ttest was used for comparison of means between two samples. One-sample t-test was used for comparison to samples arbitrarily fixed to 1 . Differences between samples were considered statistically significant for $p$ value $<0.05$. 


\section{RESULTS}

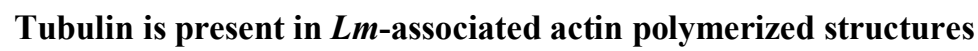

The proteomic analysis of $\mathrm{Lm}$ comet tails unexpectedly revealed that $5 \%$ of the total protein mass in these structures corresponds to $\alpha, \beta$ tubulin [20]. Taking this into account, we investigated whether $L m$ would usurp or interfere with the host tubulin cytoskeleton during intracytoplasmic life. Human intestinal epithelial Caco-2 cells were infected with GFP-expressing wild type (WT) $L m$ EGDe for $7 \mathrm{~h}$ to ensure that bacteria escape the internalization vacuole and reach the host cell cytoplasm [10]. Cells were processed for immunofluorescence and stained for tubulin detection. As compared to non-infected cells, the dense meshwork of MTs was not perturbed by bacteria and no particular rearrangement of the MT pattern in close proximity to $\mathrm{Lm}$ was detected

(Fig. 1a, NI and Inf), suggesting the absence of interplay between Lm and MTs. However, MT meshwork is composed by individual MTs with different stabilities, which are not distinguishable by tubulin labelling and their high density may limit the detection of possibly modest $L m$-induced alterations. To better assess possible alterations in the tubulin meshwork upon infection, we performed cold stability assays, wherein cold shock treatment of cells promotes depolymerization of labile MTs, allowing the observation of the most stable tubulin structures. As expected, following cold shock, most of the MTs collapsed and the density of the tubulin labelling was greatly decreased (Fig. 1a, Inf + cold shock). Surprisingly, we detected a tubulin enrichment at one pole of some bacteria strongly resembling the well described $\mathrm{Lm}$ actin comet tail [33] (Fig. 1a, Inf + cold shock). We also analyzed infected cells undergoing mitosis, in which the formation of the mitotic spindle extensively remodels the MT meshwork, resulting in a decreased MT density, especially at the cell cortex. In these cells, tubulin-enriched structures resembling comet tails were detected even without cold shock (Fig. 1a, Inf Mitotic). This suggests that, besides its well characterized usurpation of the actin cytoskeleton, $L m$ would be able to interfere with the tubulin cytoskeleton to form comet-like tubulin structures.

To investigate whether tubulin enrichments correlated with $\mathrm{Lm}$-driven actin polymerization, infected cells were simultaneously labelled for tubulin and actin. We detected tubulin enrichments associated to both $\mathrm{Lm}$-induced actin comets and clouds surrounding bacteria (Fig. 1b). Quantification of these events showed that $62.0 \pm 5.1 \%$ of the actin comet tails were positive for tubulin, while only $25.3 \pm 3.5 \%$ of the $L m$-actin clouds displayed detectable tubulin levels (Fig. 1c). Events of tubulin accumulation at the vicinity of $L m$ without detectable levels of actin were extremely rare $(<2 \%)$. These data indicate that the accumulation of tubulin at $L m$ vicinity largely correlates with the existence of polymerized actin at those sites. To further corroborate this observation, we assessed whether the isogenic $L m$ mutant $\triangle a c t A$, unable to polymerize actin, would accumulate tubulin in its

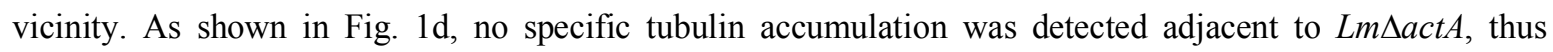
reinforcing the requirement for ActA or ActA-induced actin filament formation for the recruitment of tubulin. We next evaluated whether the $L m$-associated tubulin enrichment was also detected during cellular infection with different WT Lm strains and occurred in cell lines from multiple origins. For that, Caco-2 cells were infected with WT Lm EGD and 10403S strains (Fig. S1a) and Lm EGDe was used to infect HeLa, BeWo, Ptk2, J774 and Jeg-3 cells (Fig. S1b). In all these conditions, we detected tubulin-enriched structures at $L m$-induced actin comet tails. In agreement with data obtained in Caco-2 cells (Fig. 1c), quantifications in HeLa and Ptk2 
cells revealed that, respectively, $56.8 \pm 7.0 \%$ and $67.4 \pm 8.2 \%$ of actin comet tails were associated with tubulin structures. The levels of tubulin association to $L m$ actin clouds were lower $(28.6 \pm 4.3 \%$ and $28.0 \pm 7.4 \%$, respectively) (Fig. S1c). Altogether, our observations indicate that tubulin is enriched in the vicinity of cytosolic $\mathrm{Lm}$ and associates with $\mathrm{Lm}$-induced actin comet tails and clouds, suggesting for the first time a role for tubulin in the $L m$ intracellular life cycle.

\section{Lm-associated tubulin structures do not behave as microtubules}

Given that $L m$-associated tubulin structures appeared to be resistant to cold shock, similarly to stable MTs, we further characterized these tubulin enrichments as compared to the well-established MT structure. In particular, given that multiple tubulin post-translational modifications (PTMs, e.g. detyrosination, acetylation, tyrosination and glutamylation) were associated to MT dynamics and stability [34], we investigated if tubulin associated to intracellular $L m$ was modified at the post-translational level. Caco-2 cells were infected with $L m$, subjected to cold shock and immunolabelled for detection of total, acetylated, detyrosinated or tyrosinated tubulin. As expected, cold stable MTs were detected by total tubulin immunolabeling as well as by PTM-specific antibodies revealing acetylated or detyrosinated modifications (Fig. 2a, arrowheads). However, while clearly detected by total tubulin staining, $L m$-associated tubulin comets were not detected by specific acetylated $(\mathrm{n}=23)$ or detyrosinated $(\mathrm{n}=40)$ tubulin labeling (Fig. 2a, arrows). In turn, tyrosinated tubulin immunolabelling revealed both MTs and soluble tubulin heterodimers as well as Lm-associated tubulin comets (Fig. 2a). These observations suggest that although $L m$-induced tubulin enrichments resist to cold shock, their stability does not rely on tubulin PTMs as in cold-stable MTs. These data raised the hypothesis that $L m$-associated tubulin comets and clouds could be structurally different from MTs. To further explore this possibility, we chemically interfered with the dynamics of MT network by incubating Lm-infected Ptk2 and Caco-2 cells with the MTdepolymerizing drugs colcemid and nocadozole, respectively, during the last $2 \mathrm{~h}$ of infection. In Caco- 2 cells, we also added nocodazole for $6 \mathrm{~h}$ after $\mathrm{Lm}$ internalization $(1 \mathrm{~h})$. Cells were then processed for immunofluorescence analysis of tubulin and actin networks. We observed that none of the drugs affected the formation of tubulin-enriched $\mathrm{Lm}$ actin comets (Fig. $2 \mathrm{~b}$ and Fig. S2). The frequency of both actin comets and clouds positive for tubulin was comparable in control and colcemid-treated Ptk2 cells (Fig. 2c). Similar results were obtained for Caco-2 cells under control or nocodazole-treated conditions (Fig. 2d). To assess whether MT depolymerization through nocodazole would affect intracellular infection, we incubated Caco-2 cells with $\mathrm{Lm}$ for $1 \mathrm{~h}$ to allow the bacteria to be internalized and then added nocodazole for $6 \mathrm{~h}$. The numbers of intracellular $L m$ were quantified at 1, 4 and $6 \mathrm{~h}$ after nocodazole addition. Both the numbers of $L m$ and its rate of intracellular replication were similar in control and nocodazole -treated conditions (Fig. 2e), indicating that intact MTs are not required for $\mathrm{Lm}$ intracellular life. Taken together, our data suggest that tubulin structures associated to $\mathrm{Lm}$ actin comets are most probably structurally different from MTs.

\section{Lm-associated tubulin coats actin comet tails}


Our observations by conventional epifluorescence microscopy (Fig. 1 and 2) suggested that tubulin associated to $L m$-induced actin polymerization exhibits a speckled pattern and is organized in a hollow tube covering the actin comet tail or displayed as an external layer covering actin clouds.

To deeply characterize tubulin association to intracellular $\mathrm{Lm}$ and its actin comet tail, we analyzed $\mathrm{Lm}$-infected HeLa cells by stimulated emission depletion (STED) super-resolution microscopy (Fig. 3). The pattern and distribution of tubulin and actin at $L m$ comet tails were analyzed, quantified and compared using fluorescence intensity profiles (RGB profiles) at specific regions of interest (ROIs). Fig. 3a shows Z-stack images of a tubulin- and actin-labeled $L m$ comet tail. While actin labelling revealed a uniform and continuous pattern along the length of the comet tail, tubulin displayed again a speckled pattern with intermittent high and low intensity regions. In agreement, RGB profiles of three different lines randomly drawn along the length of the comet tail showed higher signal variation for tubulin than for actin (Fig. 3b). In addition, RGB profiles randomly drawn perpendicularly to the tail orientation in the central plane of Z-stack progressions (Fig. 3c and d), showed that the maximum intensity of actin staining is confined within two distinct peaks of tubulin labeling located at the borders of the comet. Together with Movie S1, these data indicate that tubulin encircles the actin comet. Similar results were observed in $\mathrm{Lm}$-induced actin clouds (Fig. 3e and f), in which tubulin labeling also encircles the actin layer around the bacterium.

\section{Stathmin recruits tubulin to the $L m$ comet tail}

Taking into account that tubulin is not always associated with $L m$-induced actin structures, that it coats actin, and that it appears to be present in patches, we postulated that other proteins might trigger the establishment of signaling platforms that would serve as intermediates for tubulin recruitment. The host proteins LaXp180 and stathmin appeared as serious candidates given that the interaction between ActA and LaXp180 was identified by two-hybrid screen and validated in vitro, and stathmin, predicted to interact with LaXp180, was detected in the vicinity of intracellular $\operatorname{Lm}[24,35,23]$. Since stathmin interacts with free $\alpha / \beta$-tubulin dimers [22,21], we hypothesized that it could be an important intermediate player in the tubulin recruitment to $L m$ actin clouds and comets. To assess this possibility, we infected Ptk2 and HeLa cells with $\mathrm{Lm}$ and evaluated the localization of stathmin in relation to intracellular bacteria by immunofluorescence. We observed that stathmin was slightly enriched in actin clouds and throughout actin comet tails (Fig. 4a), with $92.4 \pm 2.7 \%$ of tubulin-positive comets and $84.6 \pm 3.8 \%$ of tubulin-positive clouds showing stathmin labeling (Fig. 4b). Among the tubulin-negative actin structures, stathmin was detected in $20.2 \pm 8.7 \%$ of comets and $22.2 \pm 7.2 \%$ of clouds. Therefore, although the presence of stathmin strongly correlates with tubulin-positive actin structures, stathmin can also be found in actin comet tails that are negative for tubulin. This suggests that stathmin may first associate with polymerized actin structures to further recruit tubulin.

To specifically address the role of stathmin on tubulin recruitment to actin structures, we interfered with its expression in HeLa cells using specific stathmin-targeting siRNAs. The efficiency of stathmin depletion was confirmed and quantified by western blotting (Fig. S3a and b). Stathmin-depleted and control cells were infected with $\mathrm{Lm}$ and processed for immunofluorescence analysis of actin, tubulin and stathmin. Actin-polymerized structures associated with $\mathrm{Lm}$ and their association with tubulin were quantified. While in control HeLa cells, 
$50.7 \pm 10.5 \%$ of the actin comets and $27.7 \pm 5.8 \%$ of clouds showed positive signal for tubulin, in stathmindepleted cells only $29.0 \pm 9.5 \%$ of the comets $(\sim 2$-fold decrease) and $7.3 \pm 2.4 \%$ of the clouds $(\sim 4$-fold decrease) showed detectable levels of tubulin (Fig. 4c). In addition, quantification of the mean fluorescence intensity (MFI) in Z-stack projections of the actin comet tails showed that, whenever detected at the comet, both stathmin and tubulin fluorescence intensities were significantly reduced in stathmin-depleted as compared to control cells (Fig. 4d).

Interestingly, super-resolution microscopy analyses revealed the recruitment of tubulin as a single spot at the front pole of $66.3 \pm 11.9 \%$ of actin-polymerizing bacteria (Fig. 3c and 4e). Moreover, besides being present throughout the comet tail (Fig. 4e), stathmin also co-localizes with the tubulin spot at the front pole in $94.4 \pm$ $9.6 \%$ of the analyzed bacteria ( $\mathrm{n}=7$ per experiment in three independent experiments).

Together, these data confirm that stathmin is required for efficient recruitment of tubulin to $L m$-associated actin structures and suggest a role for stathmin in infection.

\section{$L m$-induced recruitment of stathmin regulates the actin comet tail}

$\mathrm{Lm}$ actin comet tails are hallmarks of a successful infection, allowing the intracellular movement of bacteria and cell-to-cell spread [11]. Actin comet tail formation is driven by the $L m$ surface protein ActA, an actinpolymerizing factor [36]. F-actin turnover is determined by the rate of incorporation versus release of actin monomers $[37,38]$ and governs the characteristics of the actin comet tail. To test whether stathmin contributes to $\mathrm{Lm}$ actin comet tail assembly and organization, control and stathmin-depleted cells were infected with $\mathrm{Lm}$ and actin comet tails were analyzed by fluorescence microscopy. Reduced stathmin expression was confirmed by western blot (Fig. S4). The MFI value for F-actin was quantified along the length of actin comet tails detected in control and stathmin-depleted cells. Under low stathmin expression, comet tail-associated actin MFI was decreased by 2 -fold as compared to control cells (Fig. 5a). In addition, quantification of total actin comet tail length showed that comets are in average 30\% longer in stathmin-depleted as compared to control cells (Fig. $5 b)$. These data suggest that stathmin modulates the length and actin density along the $\mathrm{Lm}$ comet tail.

Interestingly, stathmin was recently reported to regulate the levels of active/inactive cofilin in cells [39]. Cofilin is particularly important in the depolymerization of $L m$-induced actin comet tails [40,41]. Additionally, increased levels of inactive cofilin were reported to impair F-actin depolymerization during $\mathrm{Lm}$ internalization [42]. Considering these observations, we hypothesized that reduced levels of stathmin may lead to increased levels of inactive cofilin, which would impair actin depolymerization, thus resulting in longer actin comets. To assess this hypothesis, we analyzed the levels of inactive cofilin (p-cofilin, phosphorylated at serine 3) associated to Lm actin comet tails in control and stathmin-depleted cells. As compared to control cells, a small but significant increase of the p-cofilin MFI was detected in $L m$ comets from stathmin-depleted cells (Fig. 5c and d). Overall, our data show that stathmin may play a role in the regulation of cofilin activity thus impacting the local density of actin and the length of $L m$ actin comet tails by interfering with actin depolymerization.

\section{Stathmin is required for efficient $L m$ intercellular spreading and survival}


Having observed that reduced stathmin expression impacts the Lm comet tail length and F-actin density, we reasoned that $\mathrm{Lm}$ ABM could be impaired under such conditions. To address this, we performed time-lapse microscopy analysis of control and stathmin-depleted HeLa cells infected with $\mathrm{Lm}$ for $7 \mathrm{~h}$ and evaluated the average $\mathrm{Lm}$ ABM speed. Actively motile bacteria were detected in both cells by phase contrast microscopy, as shown by arrows in the selected frames depicted in Fig. 6a (and movies S2 and S3). Lm speed in control cells was $0.16 \pm 0.02 \mu \mathrm{m} / \mathrm{sec}$, which is in line with other studies [18,43], and decreased by 2 -fold $(0.07 \pm 0.01 \mu \mathrm{m} / \mathrm{sec})$ in stathmin-depleted cells (Fig. 6b and Table S1). Given the importance of intracellular movement to $\mathrm{Lm}$ dissemination, we wondered whether reduced $L m$ intracellular speed observed in stathmin-depleted cells would impact intercellular spreading. We evaluated $L m$ infection foci area in control and stathmin-depleted conditions by immunofluorescence microscopy. Monolayers of HeLa cells were infected with $\mathrm{Lm}$ for $24 \mathrm{~h}$ and the area of regions with dense $L m$ staining (infection foci) was quantified (Fig. 6c). The infection foci area was significantly reduced in stathmin-depleted as compared to control cells (Fig. 6d and Table S2), suggesting a role for stathmin in $\mathrm{Lm}$ intercellular spread. To confirm this, control and stathmin-depleted cells were infected for $24 \mathrm{~h}$ and intracellular bacterial numbers were assessed. The bacterial load retrieved from stathmin-depleted cells was $40 \%$ lower than that recovered from control cells (Fig. 6e).

Altogether, these data show that stathmin recruitment to the $\mathrm{Lm}$-induced actin comet allows the recruitment of tubulin and the modulation of actin dynamics in the comet, which appear critical for $L m$ intracellular movement, cell-to-cell spreading and, ultimately, dissemination of the infection (Fig. 7). 


\section{DISCUSSION}

$L m$ usurpation of the host actin cytoskeleton has been studied for more than 20 years [10]. However, no robust evidence exists concerning the interplay of $L m$ with the tubulin cytoskeleton and its possible impact in infection. Here we demonstrate that tubulin is recruited to $L m$-induced actin structures via stathmin, which in turn favors bacterial intracellular motility, dissemination and, ultimately, infection.

While tubulin was suggested to be present in the actin comet tail [44], and reports have provided biochemical evidence for its presence in vitro [20], the formal evidence of the presence of tubulin in Lm-induced actin comet during cellular infection was never demonstrated. Here, we envisaged two strategies to reveal hidden tubulinrich structures associated with $L m$ underneath the dense and intricate MT meshwork spanning the cytoplasm. One explored the temperature-sensitive nature of MTs to collapse the most labile ones, exposing $L m$ tubulin comets and clouds. The other relied on the naturally occurring MT remodeling required for mitotic spindle assembly, although in this condition, tubulin comet detection is rather difficult due to (low) mitotic rates and to the impairment of actin comet formation during mitosis [45].

Our data clearly show that the $L m$-associated tubulin structures are not bona-fide MTs, which is in agreement with previous analyses which failed to demonstrate the presence of MTs together with microfilaments in Lmpolymerized actin structures [19]. Thus, to our knowledge, this is the first report of a pathogen hijacking nonpolymerized forms of cytoskeletal components during cellular infection.

Our observation that $\mathrm{Lm}$ actin clouds, but mostly actin comets, display tubulin accumulation was suggestive of a spatio-temporal regulation, possibly a sequential recruitment of tubulin to the vicinity of $L m$. Our targeted search identified stathmin as the upstream mediator of tubulin sequestering to $L m$-polymerized actin structures and unveiled its impact in actin comet tail length and F-actin density possibly via regulation of cofilin activity. Cofilin is an essential component of the $\mathrm{Lm}$ comet tail, balancing cycles of actin polymerization and depolymerization according to its own phosphorylation status [46]. In particular, phosphorylation on serine 3 renders cofilin inactive, an event already reported to control $\mathrm{Lm}$ infection at the internalization level [42]. The slight increased levels of serine 3-phosphorylated cofilin along the comet tails detected in stathmin-depleted cells, suggest an impairment in F-actin depolymerization eventually leading to longer comet tails. Similarly, others have shown that longer comet tails appear upon immunodepletion of cofilin although not leading to decreased actin density [40]. Stathmin depletion was already demonstrated to increase phospho-cofilin levels via Rho/ROCK signaling pathway independently of LIM kinases [39]. However, LIM kinases modulate Lm comet tail length and are the primary enzymes to phosphorylate cofilin, via two main pathways: Rho/ROCK and Cdc42/Rac-PAK $[42,46]$. Therefore, actin dynamics regulation by stathmin at the comet tail warrants further studies as several layers of regulation might be taking place.

Overall, we demonstrate in an unprecedent way that a MT modulator - stathmin - has the capacity to regulate actin dynamics, at least at the $\mathrm{Lm}$ comet tail, highlighting another level of interconnection between different cytoskeleton machineries.

The actin comet tail is a hallmark of bacterial intracellular movement [2]. Investigation of the specific involvement of tubulin in $\mathrm{Lm} \mathrm{ABM}$ is technically limited by the fact that depleting tubulin is not a feasible 
approach and the widely used drugs only target tubulin incorporated in MTs. Therefore, to exclude tubulin from $L m$ comet tails, the upstream recruiter - stathmin - was depleted, impairing $L m$ intracellular motility and consequently its intercellular dissemination. Stathmin sequesters free $\alpha / \beta$ heterodimeric tubulin and reduces their availability to be incorporated in MTs [21,22]. Thus, one cannot exclude the possibility that perturbing stathmin would increase the density and/or rigidity of the MT meshwork surrounding the bacterium, thus interfering with $L m$ motility. Nevertheless, we and others have observed that stathmin depletion causes only minor changes in MT density [39]. Additionally, pushing the MT dynamics towards MT formation, by using taxol, even rescued $L m$ speed impairment caused by dynamin-2 depletion and had no substantial effect on the speed of Shigella, another ABM-associated pathogen [47,48].

An alternative way to elucidate the specific involvement of tubulin in $\mathrm{Lm}$ infection would be to identify the bacterial virulence factor(s) responsible for stathmin recruitment to the $L m$-associated actin structures. ActA has been already suggested, as it was identified in a two-hybrid screen to interact with the host protein LaXp180, which in turn is predicted to interact with stathmin $[23,35]$. Nevertheless, this seems unlikely as ActA mainly accumulates at the old (posterior) bacterial pole to promote unidirectional movement $[36,49,50]$, and we clearly show that both stathmin and tubulin are present along the entire comet length as well as at the front (anterior) pole of bacteria polymerizing actin comets. Moreover, others also detected stathmin as distinct spots in a similar location in motile $\mathrm{Lm}$ [24]. Since stathmin sequestering of tubulin leads to depolymerization of MTs, stathmin localization at the bacterial front pole may reduce physical constraints and facilitate $\mathrm{Lm}$ movement, as suggested by others [47]. In our experiments, cytosolic $L m \Delta a c t A$ did not show any tubulin accumulation at its surface. We thus propose that another unknown bacterial factor could be contributing, together with ActA, to stathmin/tubulin recruitment to $L m$-associated actin structures.

Tubulin appears to accumulate as an outer layer surrounding $L m$-induced actin clouds and comet tails. The biological significance of this observation remains unknown, however it might relate with optimal bacterial spreading and survival observed when compared to comets and clouds devoid of stathmin/tubulin. One might hypothesize that such particular architecture acts as a shield with a bi-functional role: towards the inside, it could promote efficient actin polymerization/depolymerization dynamics, and towards the outside, create a MTfree space, reducing cytoplasmic restriction against $L m$ movement. Similar mechanisms were proposed for other intracellular bacterial pathogens: Shigella creates a tunnel as it destroys MT meshwork upon passage [48] and CopN virulence factor from Chlamydia inhibits MT polymerization by sequestering tubulin heterodimers, in a mechanism similar to stathmin [51].

The molecular and cellular events here described would benefit from further validation in vivo using relevant animal models (e.g. stathmin KO mice) and possibly other cytoplasm-invading pathogens (e.g. Shigella flexneri, Burkholderia pseudomallei, Mycobacterium marinum and Rickettsia ricketsiae). Although there is still much to understand concerning stathmin/tubulin function in the context of infection, this work sheds some light into an unexpected mode of cytoskeletal usurpation by pathogens and certainly paved the way for future development of novel hypothesis. 


\section{ACKNOWLEDGMENTS}

This work received funding from Norte-01-0145-FEDER-000012 - Structured program on bioengineered therapies for infectious diseases and tissue regeneration, supported by Norte Portugal Regional Operational Programme (NORTE 2020), under the PORTUGAL 2020 Partnership Agreement, through the European Regional Development Fund (FEDER). ACC and FC were supported by a Fundação para a Ciência e Tecnologia (FCT) Post-doctoral Fellowship (SFRH/BPD/88769/2012) and PhD fellowship (SFRH/BD/61825/2009), respectively, through FCT/MEC co-funded by QREN and POPH (Programa Operacional Potencial Humano). SS was supported by FCT Investigator program (COMPETE, POPH, and FCT). We thank ALM unit from IBMC/i3S for technical support, B. Fonseca (FFUP) for the BeWo cell line and H. Maiato, C. Sunkel and J. B. Relvas laboratories (IBMC/i3S) for sharing reagents. We are also thankful to J. Ferreira and A. Pereira for fruitful discussions.

\section{AUTHOR CONTRIBUTIONS}

ACC, DC and SS conceived and designed the experiments; ACC and FC performed the experiments; ACC, FC, DC and SS analyzed the data; DC and SS obtained the funding; ACC and SS wrote the manuscript. 


\section{REFERENCES}

1. Haglund CM, Welch MD (2011) Pathogens and polymers: microbe-host interactions illuminate the cytoskeleton. J Cell Biol 195 (1):7-17. doi:10.1083/jcb.201103148

2. Welch MD, Way M (2013) Arp2/3-mediated actin-based motility: a tail of pathogen abuse. Cell host \& microbe 14 (3):242-255. doi:10.1016/j.chom.2013.08.011

3. Radhakrishnan GK, Splitter GA (2012) Modulation of host microtubule dynamics by pathogenic bacteria. Biomol Concepts 3 (6):571-580. doi:10.1515/bmc-2012-0030

4. Geisler F, Leube RE (2016) Epithelial Intermediate Filaments: Guardians against Microbial Infection? Cells 5 (3):29. doi:10.3390/cells5030029

5. Torraca V, Mostowy S (2016) Septins and Bacterial Infection. Frontiers in Cell and Developmental Biology 4 (127). doi:10.3389/fcell.2016.00127

6. Rodriguez OC, Schaefer AW, Mandato CA, Forscher P, Bement WM, Waterman-Storer CM (2003) Conserved microtubule-actin interactions in cell movement and morphogenesis. Nat Cell Biol 5 (7):599-609. doi:10.1038/ncb0703-599

7. Ray K, Marteyn B, Sansonetti PJ, Tang CM (2009) Life on the inside: the intracellular lifestyle of cytosolic bacteria. Nat Rev Microbiol 7 (5):333-340. doi:10.1038/nrmicro2112

8. Radoshevich L, Cossart P (2018) Listeria monocytogenes: towards a complete picture of its physiology and pathogenesis. Nat Rev Microbiol 16 (1):32-46. doi:10.1038/nrmicro.2017.126

9. Welch MD, Rosenblatt J, Skoble J, Portnoy DA, Mitchison TJ (1998) Interaction of Human Arp2/3 Complex and the $<\mathrm{em}>$ Listeria monocytogenes $</ \mathrm{em}>$ ActA Protein in Actin Filament Nucleation. Science 281 (5373):105-108. doi:10.1126/science.281.5373.105

10. Tilney LG, Portnoy DA (1989) Actin filaments and the growth, movement, and spread of the intracellular bacterial parasite, Listeria monocytogenes. J Cell Biol 109 (4 Pt 1):1597-1608

11. Lambrechts A, Gevaert K, Cossart P, Vandekerckhove J, Van Troys M (2008) Listeria comet tails: the actinbased motility machinery at work. Trends Cell Biol 18 (5):220-227. doi:10.1016/j.tcb.2008.03.001

12. Loisel TP, Boujemaa R, Pantaloni D, Carlier MF (1999) Reconstitution of actin-based motility of Listeria and Shigella using pure proteins. Nature 401 (6753):613-616. doi:10.1038/44183

13. Wang J, King JE, Goldrick M, Lowe M, Gertler FB, Roberts IS (2015) Lamellipodin Is Important for Cellto-Cell Spread and Actin-Based Motility in Listeria monocytogenes. Infect Immun 83 (9):3740-3748. doi:10.1128/IAI.00193-15

14. Fattouh R, Kwon H, Czuczman MA, Copeland JW, Pelletier L, Quinlan ME, Muise AM, Higgins DE, Brumell JH (2015) The Diaphanous-Related Formins Promote Protrusion Formation and Cell-to-Cell Spread of Listeria monocytogenes. The Journal of Infectious Diseases 211 (7):1185-1195. doi:10.1093/infdis/jiu546

15. Greiffenberg L, Goebel W, Kim KS, Weiglein I, Bubert A, Engelbrecht F, Stins M, Kuhn M (1998) Interaction of Listeria monocytogenes with human brain microvascular endothelial cells: InlB-dependent invasion, long-term intracellular growth, and spread from macrophages to endothelial cells. Infect Immun 66 (11):5260-5267

16. Kuhn M (1998) The microtubule depolymerizing drugs nocodazole and colchicine inhibit the uptake of Listeria monocytogenes by P388D1 macrophages. FEMS Microbiol Lett 160 (1):87-90. doi:S03781097(98)00017-2

17. Guzman CA, Rohde M, Chakraborty T, Domann E, Hudel M, Wehland J, Timmis KN (1995) Interaction of Listeria monocytogenes with mouse dendritic cells. Infect Immun 63 (9):3665-3673

18. Lacayo CI, Theriot JA (2004) Listeria monocytogenes actin-based motility varies depending on subcellular location: a kinematic probe for cytoarchitecture. Mol Biol Cell 15 (5):2164-2175. doi:10.1091/mbc.E03-100747 
19. Mounier J, Ryter A, Coquis-Rondon M, Sansonetti PJ (1990) Intracellular and cell-to-cell spread of Listeria monocytogenes involves interaction with F-actin in the enterocytelike cell line Caco-2. Infect Immun 58 (4):1048-1058

20. Van Troys M, Lambrechts A, David V, Demol H, Puype M, Pizarro-Cerda J, Gevaert K, Cossart P, Vandekerckhove J (2008) The actin propulsive machinery: the proteome of Listeria monocytogenes tails. Biochem Biophys Res Commun 375 (2):194-199. doi:10.1016/j.bbrc.2008.07.152

21. Gavet O, Ozon S, Manceau V, Lawler S, Curmi P, Sobel A (1998) The stathmin phosphoprotein family: intracellular localization and effects on the microtubule network. J Cell Sci 111 ( Pt 22):3333-3346

22. Gupta KK, Li C, Duan A, Alberico EO, Kim OV, Alber MS, Goodson HV (2013) Mechanism for the catastrophe-promoting activity of the microtubule destabilizer Op18/stathmin. Proc Natl Acad Sci U S A 110 (51):20449-20454. doi:10.1073/pnas.1309958110

23. Maucuer A, Camonis JH, Sobel A (1995) Stathmin interaction with a putative kinase and coiled-coilforming protein domains. Proc Natl Acad Sci U S A 92 (8):3100-3104

24. Pfeuffer T, Goebel W, Laubinger J, Bachmann M, Kuhn M (2000) LaXp180, a mammalian ActA-binding protein, identified with the yeast two-hybrid system, co-localizes with intracellular Listeria monocytogenes. Cell Microbiol 2 (2):101-114. doi:cmi34 []

25. Glaser P, Frangeul L, Buchrieser C, Rusniok C, Amend A, Baquero F, Berche P, Bloecker H, Brandt P, Chakraborty T, Charbit A, Chetouani F, Couvé E, de Daruvar A, Dehoux P, Domann E, Domínguez-Bernal G, Duchaud E, Durant L, Dussurget O, Entian K-D, Fsihi H, Portillo FG-D, Garrido P, Gautier L, Goebel W, Gómez-López N, Hain T, Hauf J, Jackson D, Jones L-M, Kaerst U, Kreft J, Kuhn M, Kunst F, Kurapkat G, Madueño E, Maitournam A, Vicente JM, Ng E, Nedjari H, Nordsiek G, Novella S, de Pablos B, Pérez-Diaz JC, Purcell R, Remmel B, Rose M, Schlueter T, Simoes N, Tierrez A, Vázquez-Boland J-A, Voss H, Wehland J, Cossart P (2001) Comparative Genomics of $<$ em $>$ Listeria $</$ em $>$ Species. Science 294 (5543):849-852. doi:10.1126/science. 1063447

26. Leitao E, Costa AC, Brito C, Costa L, Pombinho R, Cabanes D, Sousa S (2014) Listeria monocytogenes induces host DNA damage and delays the host cell cycle to promote infection. Cell Cycle 13 (6):928-940. doi: $10.4161 /$ cc. 27780

27. Mengaud J, Geoffroy C, Cossart P (1991) Identification of a new operon involved in Listeria monocytogenes virulence: its first gene encodes a protein homologous to bacterial metalloproteases. Infection and Immunity 59 (3):1043-1049

28. Portnoy DA, Jacks PS, Hinrichs DJ (1988) Role of hemolysin for the intracellular growth of Listeria monocytogenes. The Journal of Experimental Medicine 167 (4):1459-1471. doi:10.1084/jem.167.4.1459

29. Schindelin J, Arganda-Carreras I, Frise E, Kaynig V, Longair M, Pietzsch T, Preibisch S, Rueden C, Saalfeld S, Schmid B, Tinevez JY, White DJ, Hartenstein V, Eliceiri K, Tomancak P, Cardona A (2012) Fiji: an opensource platform for biological-image analysis. Nat Methods 9 (7):676-682. doi:nmeth.2019

30. Schneider CA, Rasband WS, Eliceiri KW (2012) NIH Image to ImageJ: 25 years of image analysis. Nat Methods 9 (7):671-675

31. Chong R, Squires R, Swiss R, Agaisse H (2011) RNAi screen reveals host cell kinases specifically involved in Listeria monocytogenes spread from cell to cell. PLoS One 6 (8):e23399. doi:10.1371/journal.pone.0023399

32. Reis O, Sousa S, Camejo A, Villiers V, Gouin E, Cossart P, Cabanes D (2010) LapB, a novel Listeria monocytogenes LPXTG surface adhesin, required for entry into eukaryotic cells and virulence. J Infect Dis 202 (4):551-562. doi:Doi 10.1086/654880

33. Shenoy VB, Tambe DT, Prasad A, Theriot JA (2007) A kinematic description of the trajectories of Listeria monocytogenes propelled by actin comet tails. Proc Natl Acad Sci U S A 104 (20):8229-8234. doi:0702454104

34. Song Y, Brady ST (2015) Post-translational modifications of tubulin: pathways to functional diversity of microtubules. Trends Cell Biol 25 (3):125-136. doi:10.1016/j.tcb.2014.10.004 
35. Bauer S, Pfeuffer T, Kuhn M (2003) Identification and characterisation of regions in the cellular protein LaXp180 and the Listeria monocytogenes surface protein ActA necessary for the interaction of the two proteins. Mol Genet Genomics 268 (5):607-617. doi:10.1007/s00438-002-0775-1

36. Rafelski SM, Theriot JA (2005) Bacterial shape and ActA distribution affect initiation of Listeria monocytogenes actin-based motility. Biophys J 89 (3):2146-2158. doi:S0006-3495(05)72858-7

37. Goldberg MB (2001) Actin-based motility of intracellular microbial pathogens. Microbiol Mol Biol Rev 65 (4):595-626, table of contents. doi:10.1128/MMBR.65.4.595-626.2001

38. Nanavati D, Ashton FT, Sanger JM, Sanger JW (1994) Dynamics of actin and alpha-actinin in the tails of Listeria monocytogenes in infected PtK2 cells. Cell Motil Cytoskeleton 28 (4):346-358. doi:10.1002/cm.970280408

39. Byrne FL, Yang L, Phillips PA, Hansford LM, Fletcher JI, Ormandy CJ, McCarroll JA, Kavallaris M (2014) RNAi-mediated stathmin suppression reduces lung metastasis in an orthotopic neuroblastoma mouse model. Oncogene 33 (7):882-890. doi:10.1038/onc.2013.11

40. Rosenblatt J, Agnew BJ, Abe H, Bamburg JR, Mitchison TJ (1997) Xenopus actin depolymerizing factor/cofilin (XAC) is responsible for the turnover of actin filaments in Listeria monocytogenes tails. J Cell Biol 136 (6):1323-1332

41. Giganti A, Plastino J, Janji B, Van Troys M, Lentz D, Ampe C, Sykes C, Friederich E (2005) Actin-filament cross-linking protein T-plastin increases Arp2/3-mediated actin-based movement. J Cell Sci 118 (Pt 6):12551265. doi:jcs. 01698

42. Bierne H, Gouin E, Roux P, Caroni P, Yin HL, Cossart P (2001) A role for cofilin and LIM kinase in Listeria-induced phagocytosis. J Cell Biol 155 (1):101-112. doi:10.1083/jcb.200104037

43. Theriot JA, Rosenblatt J, Portnoy DA, Goldschmidt-Clermont PJ, Mitchison TJ (1994) Involvement of profilin in the actin-based motility of L. monocytogenes in cells and in cell-free extracts. Cell 76 (3):505-517. doi:0092-8674(94)90114-7

44. Buchwalow IB, Emoto M, Brich M, Kaufmann SHE (1997) Involvement of tubulin and inhibitory G proteins in the interaction of Listeria monocytogenes with mouse hepatocytes. Infection and Immunity 65 (3):1095-1097

45. Sanger JM, Sanger JW (2012) Insights into cell division using Listeria monocytogenes infections of PtK2 renal epithelial cells. Cytoskeleton (Hoboken) 69 (11):992-999. doi:10.1002/cm.21076

46. Zheng K, Kitazato K, Wang Y, He Z (2016) Pathogenic microbes manipulate cofilin activity to subvert actin cytoskeleton. Crit Rev Microbiol 42 (5):677-695. doi:10.3109/1040841X.2015.1010139

47. Henmi Y, Tanabe K, Takei K (2011) Disruption of microtubule network rescues aberrant actin comets in dynamin2-depleted cells. PLoS One 6 (12):e28603. doi:10.1371/journal.pone.0028603

48. Yoshida S, Handa Y, Suzuki T, Ogawa M, Suzuki M, Tamai A, Abe A, Katayama E, Sasakawa C (2006) Microtubule-severing activity of Shigella is pivotal for intercellular spreading. Science 314 (5801):985-989. doi:314/5801/985

49. Kocks C, Hellio R, Gounon P, Ohayon H, Cossart P (1993) Polarized distribution of Listeria monocytogenes surface protein ActA at the site of directional actin assembly. J Cell Sci 105 ( Pt 3):699-710

50. Rafelski SM, Theriot JA (2006) Mechanism of polarization of Listeria monocytogenes surface protein ActA. Mol Microbiol 59 (4):1262-1279. doi:MMI5025

51. Archuleta TL, Du Y, English CA, Lory S, Lesser C, Ohi MD, Ohi R, Spiller BW (2011) The Chlamydia effector chlamydial outer protein $\mathrm{N}(\mathrm{CopN})$ sequesters tubulin and prevents microtubule assembly. J Biol Chem 286 (39):33992-33998. doi:10.1074/jbc.M111.258426 
Manuscript Costa et al. 


\section{FIGURE LEGENDS}

\section{Figure 1}

Tubulin enrichments are detected in actin structures polymerized by $\mathbf{L m}$. (a) Caco-2 cells non-infected (NI) or infected with $L m$ expressing GFP (Inf; MOI 30 for $7 \mathrm{~h}$ ) were fixed at $37^{\circ} \mathrm{C}$ (NI, Inf and Mitotic Inf) or subjected to cold shock $\left(10 \mathrm{~min}\right.$ at $\left.4{ }^{\circ} \mathrm{C}\right)$ prior to room temperature fixation (Inf + cold shock). Micrographs show representative deconvoluted Z-stack projections. Insets are shown as high-magnification composite (merge) and single-channel images displaying Lm (GFP, green) and tubulin (red) labeling. DNA was labeled with DAPI (blue). (b) Microscopy images of Caco-2 cells infected with wild type (WT) Lm (MOI 30 for 7 h) subjected to cold shock before fixation. Insets are shown as single-channel images displaying tubulin (red) accumulation at actin clouds and comet tails (green) associated to $\mathrm{Lm}$. (c) Percentage of $\mathrm{Lm}$ actin comets or clouds showing tubulin accumulation as quantified from (b). Results are mean \pm SEM of three independent experiments $(\mathrm{n} \sim 100$ actin comets or clouds per experiment). (d) Micrographs of Caco-2 cells infected with Lm $\Delta a c t A$ mutant (MOI 30 for $7 \mathrm{~h}$ ) subjected to cold shock before fixation. Insets are shown as highmagnification composite (merge) and single-channel images displaying tubulin (red) and $L m$ (green). Scale bars correspond to $10 \mu \mathrm{m}$.

\section{Figure 2}

$\mathrm{Lm}$-associated tubulin structures do not behave as microtubules. (a) Caco-2 cells infected with WT $\mathrm{Lm}$ (MOI 30 for $7 \mathrm{~h}$ ) subjected to cold shock before fixation. Cells were immunolabelled for tubulin (red) and for specific tubulin post-translational modifications (PTM, green): acetylation, detyrosination or tyrosination. Micrographs shown are deconvoluted versions of multiple Z-planes projected into a single image. Magnified versions of the insets are shown as single channel images. Arrows indicate $L m$-associated tubulin structures. Arrowheads show acetylated or detyrosinated tubulin incorporated in stable microtubules. (b to d) Infected Ptk2 (MOI 50 for $7 \mathrm{~h}$ ) and Caco-2 cells (MOI 30 for $7 \mathrm{~h}$ ) were treated with drugs inhibiting microtubule formation, respectively colcemid and nocodazole. Colcemid $(0.27 \mu \mathrm{M})$ was added to Ptk2-infected cells during the last $2 \mathrm{~h}$ of infection. Nocodazole $(33 \mu \mathrm{M})$ was added to Caco-2-infected cells during the last $2 \mathrm{~h}$ of infection or for $6 \mathrm{~h}$ starting after $1 \mathrm{~h} \mathrm{Lm}$ internalization step (b) Cells were fixed, labeled for tubulin (red), actin (green) and DNA (blue), analyzed by fluorescence microscopy. Micrographs are Z axis projections. Quantification of the percentage of $L m$ showing tubulin accumulation together with actin comets or clouds in (c) untreated or colcemid treated Ptk2 cells, and in (d) untreated or nocodazole treated Caco-2 cells. (e) Quantification of intracellular $L m$ by CFU counting. Caco-2 cells were incubated with Lm for $1 \mathrm{~h}$ and incubated with nocodazole (33 $\mu \mathrm{M}$, Noco). Intracellular bacteria were quantified at 1, 4 and $6 \mathrm{~h}$ post nocodazole incubation and compared with control conditions. Results are mean \pm SEM of three independent experiments, at least 100 structures were analyzed per condition in each experiment. Scale bars correspond to $5 \mu \mathrm{m}$ in Ptk2 images and $10 \mu \mathrm{m}$ in Caco-2 images. 


\section{Figure 3}

$\boldsymbol{L m}$-associated tubulin coats actin comet tails. (a to f) HeLa cells were infected with WT Lm (MOI 50 for 7 h) and subjected to cold shock before processing for super-resolution microscopy (STED). (a and c) Representative images of $Z$ axis progressions depicting tubulin (red) and actin (SiRactin, green) labeling at the $L m$ comet tail. (b and d) Fluorescence intensity profiles of tubulin (red) and actin (green) of ROIs indicated as white dashed lines at intermediate planes Z2 in (a) and Z3 in (c). (e) Composite and single-channel images of $\mathrm{Lm}$-associated actin clouds labeled for tubulin (red) and actin (green). (f) Fluorescence intensity profiles of ROIs 1 and 2 indicated as white dashed lines in (e). All plots and images are representative of three independent experiments. Black dashed lines in the plots indicate the fluorescence intensity peaks. Scale bars correspond to (a and c) $1 \mu \mathrm{m}$ and (e) $0.5 \mu \mathrm{m}$.

\section{Figure 4}

Stathmin recruits tubulin to the $\boldsymbol{L m}$ comet tail. (a) Maximum intensity projection of deconvoluted images showing Ptk2 and HeLa cells infected with $\mathrm{Lm}$ for $7 \mathrm{~h}$ with MOI 50. Upon cold shock, cells were labeled for stathmin, tubulin, actin and DNA. Higher magnification insets are shown as single-channel images. For each cell line, upper panels highlight clouds and lower panels highlight comets. (b) Quantification of stathmin recruitment to $L m$-associated actin structures, either positive or negative for tubulin. Results are mean \pm SEM from five independent experiments, with $\sim 150$ actin structures analyzed per experiment. (c) Quantification of the percentage of $\mathrm{Lm}$ actin comets or clouds showing tubulin accumulation in control (siC) and stathmindepleted HeLa cells (siStat). Results are mean \pm SD from four independent experiments, at least 100 actin structures were analyzed per condition in each experiment. (d) Fold change of mean fluorescence intensity (MFI) for stathmin and tubulin along $\mathrm{Lm}$ actin comet tails, in control (siC) and stathmin-depleted HeLa cells (siStat). Values for siC cells were normalized to 1 and data for siStat cells are represented as relative values. Results are mean \pm SD from three independent experiments, at least 40 comets were analyzed per condition in each experiment. (e) Super-resolution single-plane micrograph of $L m$ with a comet tail within an infected HeLa cell (7 h post-infection). Merge image shows immunostaining for stathmin (green) and tubulin (red), and wheat germ agglutinin (WGA, cyan) labeling of the bacterium. Asterisks indicate statistical significance as determined by Student $t$ test: $p$ value $<0.05(*),<0.01(* *)$. Scale bar represents (a) $10 \mu \mathrm{m}$ and (e) $0.5 \mu \mathrm{m}$.

\section{Figure 5}

Stathmin modulates actin dynamics at the comet tail. (a to d) Control ( $\mathrm{siC}$ ) and stathmin-depleted (siStat) HeLa cells were infected for $7 \mathrm{~h}$ with $\mathrm{Lm}$ and processed for immunofluorescence analysis. (a) Quantification of actin MFI along the comet tails. Values for siC cells were normalized to 1 and data in siStat cells are represented as relative values. Results are mean $\pm \mathrm{SD}$ of four independent experiments, at least 40 comets were 
analyzed per condition in each experiment. (b) Quantification of actin comet tail length. Each data point represents one comet tail and horizontal black lines are mean \pm SEM from four independent experiments. (c) Z-stack projection of images from cells labeled for DNA (blue), actin (red) and phospho-S3-cofilin (green). Scale bar represents $2 \mu \mathrm{m}$. (d) Fold change of MFI for phosphorylated (inactive) cofilin, normalized to actin levels, in $\mathrm{Lm}$-induced comet tails. Results are mean $\pm \mathrm{SD}$ of three independent experiments, at least X 50 comets were analyzed per experiment. Asterisks indicate statistical significance as determined by Student $t$ test: $p<$ $0.05(*), p<0.0001(* * *)$.

\section{Figure 6}

Stathmin is required for efficient $\boldsymbol{L} \boldsymbol{m}$ intercellular spreading. (a to e) HeLa cells were transfected with control (siC) or stathmin (siStat) siRNA and infected with Lm. (a) Sequential phase contrast frames of timelapse microscopy performed within the time window of 7 to $14 \mathrm{~h}$ post-infection. Arrows indicate a single bacterium actively polymerizing an actin tail. The black line drawn in the last frame $(680 \mathrm{sec})$ shows the path defined by bacterial movement over time. (b) Quantification of the average speed of $L m$ in siC and siStat cells. Each data point represents one bacterium and horizontal bars are mean $\pm \mathrm{SEM}$ of four independent experiments. (c and d) Confluent monolayers of $\mathrm{siC}$ and siStat HeLa cells were infected for $24 \mathrm{~h}$ with MOI 0.5 and immunolabeled to detect $\mathrm{Lm}$. (c) Immunofluorescence images show infection foci areas bordered by manually drawn white dash lines. Scale bar represents $50 \mu \mathrm{m}$. (d) Quantification of the infection foci area. Each dot corresponds to a focus of infection and horizontal bars are mean \pm SEM of three independent experiments. (e) siC and siStat HeLa cells were infected for $24 \mathrm{~h}$, lysed and $\mathrm{Lm}$ intracellular numbers were determined by CFU counting. Relative CFU values are shown and are mean \pm SD of four independent experiments. Asterisks indicate statistical significance as determined by Student $t$ test: $p<0.01(* *), p<0.0001(* * *)$.

\section{Figure 7}

Proposed model for the role of stathmin/tubulin in $\mathbf{L m}$ infection. $L m$ recruits stathmin, which sequesters tubulin, creating an external layer surrounding actin comet tails. This arrangement promotes optimized actin dynamics, bacterial propulsion and efficient dissemination, ultimately favoring $\mathrm{Lm}$ infection. Stathmin abrogation from cells leads to increased levels of inactive cofilin in the actin comet, decreased amounts of Fand increased comet tail length. Consequently, $L m$ speed is reduced, bacterial spreading is impaired, and bacterial loads are diminished.

\section{Figure S1 (relative to Figure 1)}

Tubulin comets are ubiquitously detected in different $\boldsymbol{L} \boldsymbol{m}$ strains and cell lines. (a to c) Cells were infected for $7 \mathrm{~h}$ and subjected to cold shock prior to fixation for immunofluorescence analysis. Representative Z-stack 
projections of deconvoluted images are shown. Insets shown as high-magnification composite (merge) and single-channel images displaying tubulin (red), actin (green) and DNA (blue). Scale bars represent $10 \mu \mathrm{m}$. (a) Caco-2 cells were infected with Lm strains EGD and 10403S. (b) Human (HeLa, Jeg-3, BeWo), murine (J774) and rat kangaroo (Ptk2) cell lines were infected with $L m$ EGDe. (c) Quantification of the percentage of $L m$ actin comets and clouds showing tubulin accumulation in HeLa and Ptk2 cells. Results are mean \pm SEM of three independent experiments ( $\mathrm{n} \sim 100$ per experiment).

\section{Figure S2 (relative to Figure 2)}

Nocodazole-treatment do not perturb the formation of $\mathbf{L m}$-associated tubulin comets. Caco-2 cells infected with $\mathrm{Lm}$. Following the bacterial internalization step (1h), cells were incubated with nocodazole (or DMSO as control) for $6 \mathrm{~h}$, subjected to cold shock, fixed and labeled for tubulin (red), actin (green) and DNA (blue) to be analyzed by fluorescence microscopy. The micrographs are $\mathrm{Z}$ axis projections of deconvolutes images. Quantification of the percentage of $\mathrm{Lm}$ showing tubulin accumulation together with actin comets is shown in Fig. 2e. Scale bars correspond to $10 \mu \mathrm{m}$.

\section{Figure S3 (relative to Figure 4)}

Efficiency of stathmin depletion in HeLa cells. (a) Western blot analysis of stathmin expression in HeLa cells treated with control (siC) or with stathmin (siStat) siRNA. GAPDH levels were used as loading control. (b) Quantification of siRNA-mediated silencing of stathmin as evaluated by densitometric analysis of Western blot bands, normalized for GAPDH expression. Results are mean \pm SD from four independent experiments. Asterisks indicate statistical significance as determined by Student $t$ test: $p<0.0001$ (****).

\section{Figure S4 (relative to Figure 5)}

Efficiency of stathmin depletion in HeLa cells. Stathmin expression levels evaluated by densitometric analysis of Western blot bands, normalized for GAPDH expression. Results are mean $\pm \mathrm{SD}$ of four independent experiments. Asterisks indicate statistical significance as determined by Student $t$ test: $p<0.01\left({ }^{* *}\right)$.

\section{Table S1 (relative to Figure 6b)}

Stathmin is required for $\mathbf{L} \boldsymbol{m}$ maximal intracellular speed. Control ( $\mathrm{siC}$ ) and stathmin-depleted (siStat) HeLa cells were infected with $\mathrm{Lm}$ and imaged by time-lapse microscopy from 7 to $14 \mathrm{~h}$ post-infection. Values for $\mathrm{Lm}$ speed are mean $\pm \mathrm{SD}$ of approximately 15 bacteria per condition, in each of the four independent experiments. Fold change (siC/siStat) and statistical significance ( $p$ value, Student $t$ test) were calculated. 
Table S2 (relative to Figure 6d)

Stathmin is required for $\mathbf{L m}$ cell-to-cell spreading. Monolayers of control (siC) and stathmin-depleted (siStat) HeLa cells were infected for $24 \mathrm{~h}$ with MOI 0.5, fixed and labeled for $\mathrm{Lm}$. Quantification of infection foci areas was performed after multiple image alignment. Values are mean $\pm \mathrm{SD}$ of at least 60 infection foci areas, per each of the three independent experiments. Fold change (siC/siStat) and statistical significance ( $p$ value, Student $t$ test) were calculated.

\section{Movie S1 (relative to Figure 3)}

Tubulin coats actin comet tails polymerized by $\mathbf{L m}$. HeLa cells infected with $L m$ were labeled for tubulin (red), $L m$ (WGA, cyan) and actin (SiRactin, green). Movie shows a $360^{\circ}$ rotation of the $L m$ comet tail obtained by three-dimensional reconstitution of stimulated emission depletion (STED) super-resolution microscopy images.

\section{Movies S2 and S3 (relative to Figure 6a, b and Table S1)}

Stathmin controls $\mathbf{L m}$ speed. Control (Movie S2) and stathmin-depleted cells (Movie S3) infected with $\mathrm{Lm}$ were subjected to phase-contrast live-imaging from 7 to $14 \mathrm{~h}$ post-infection. Arrows indicate the position of $\mathrm{Lm}$ throughout time and the black line shows the described path. Videos are reproduced at 5 frames per second (AVI). 
a
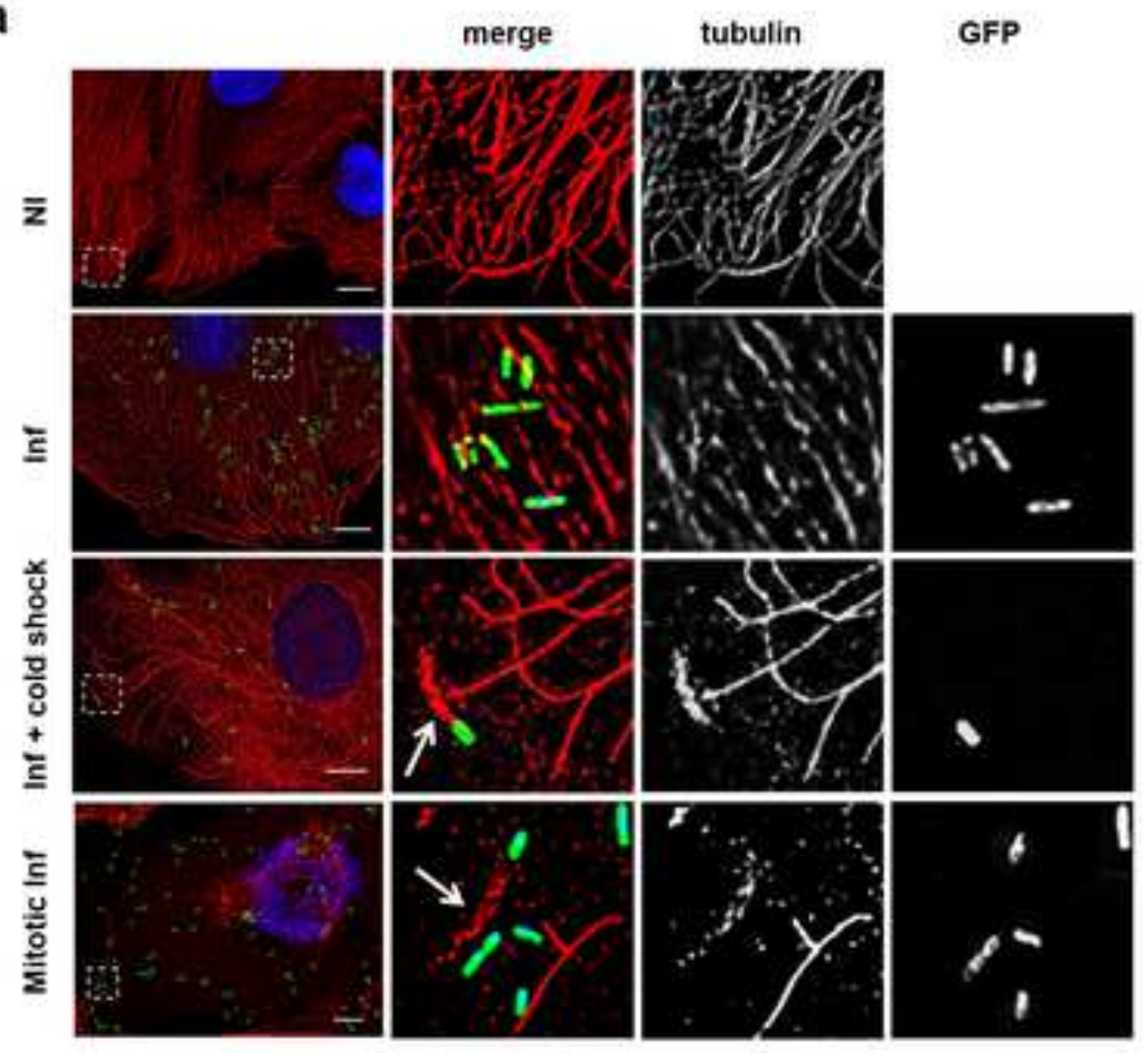

d

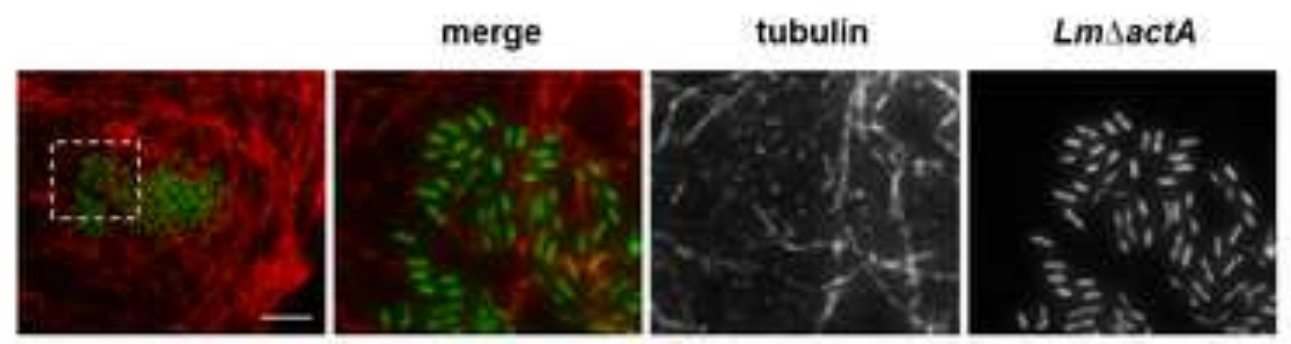

b

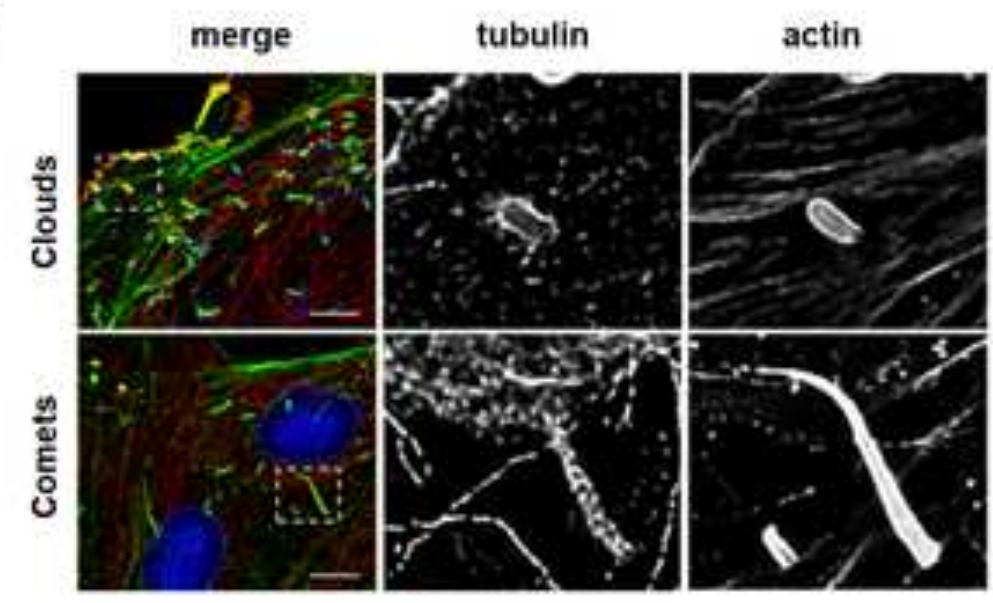

C

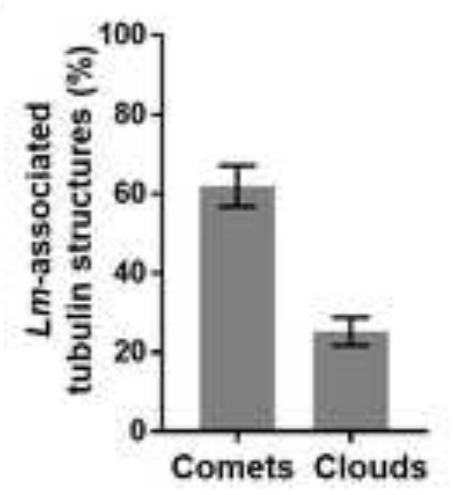


a

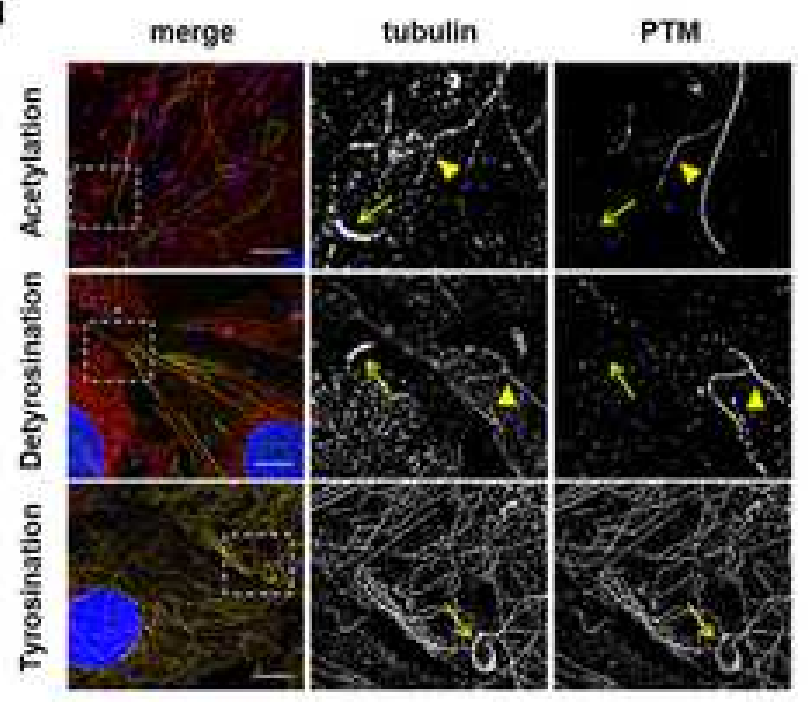

b

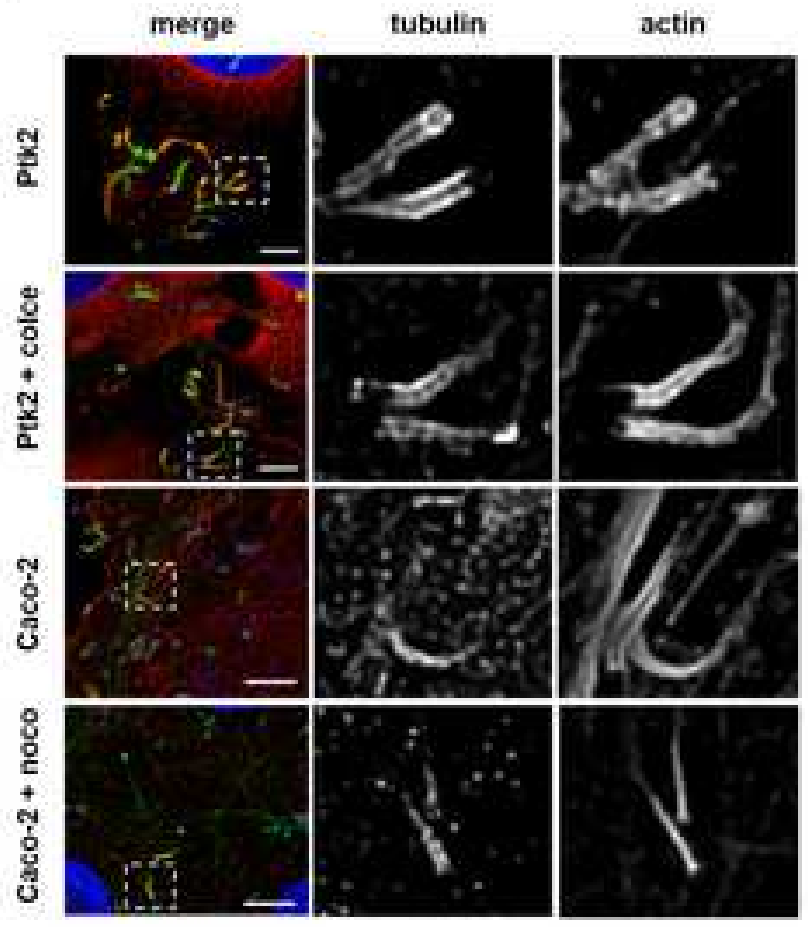

C

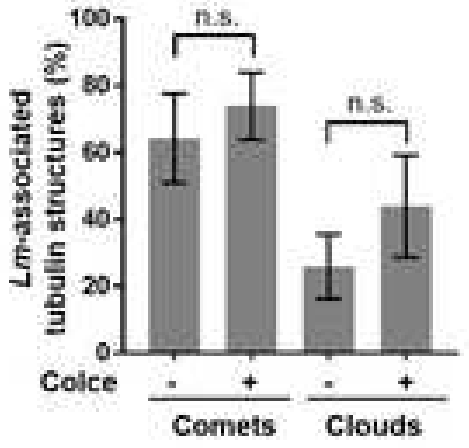

d

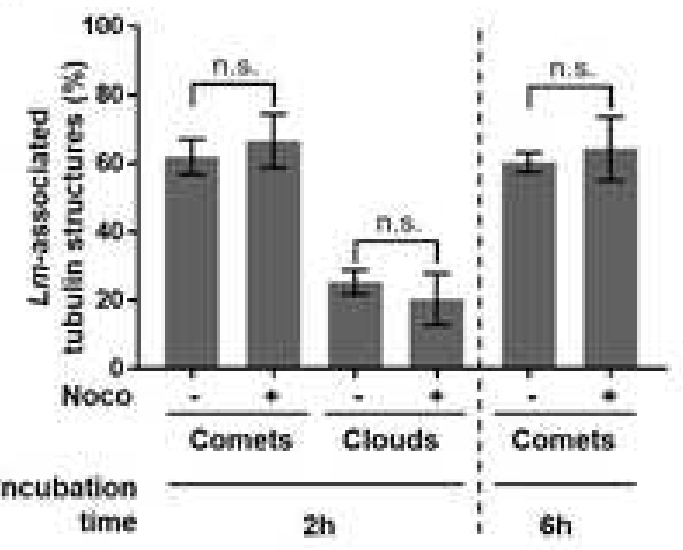

e

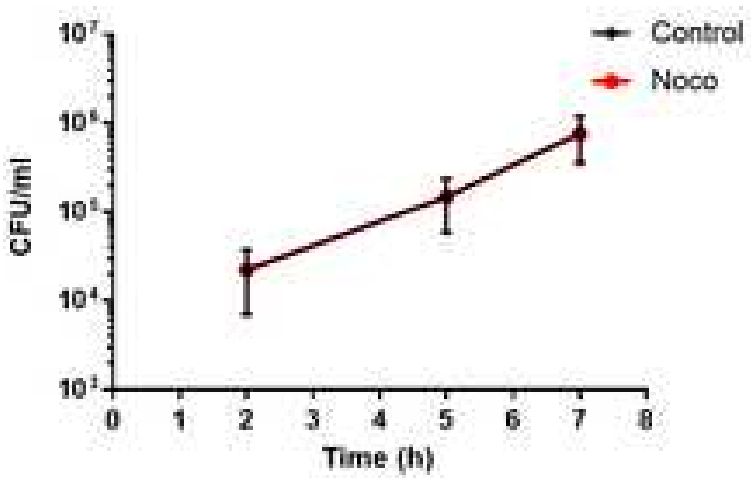


a

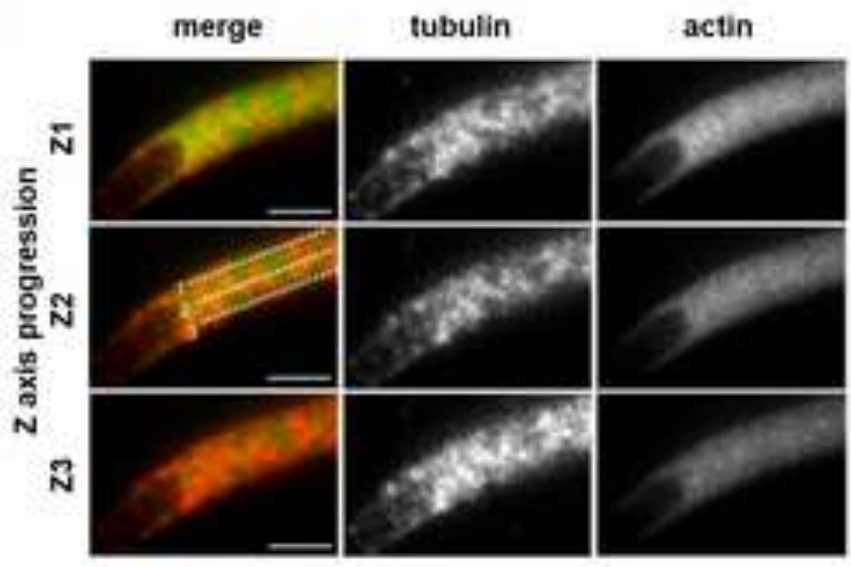

C

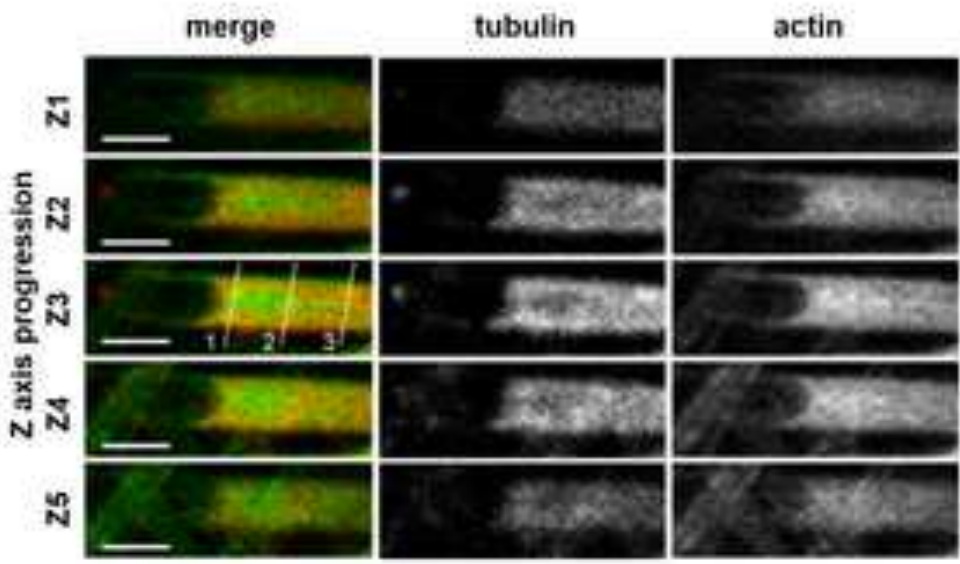

e
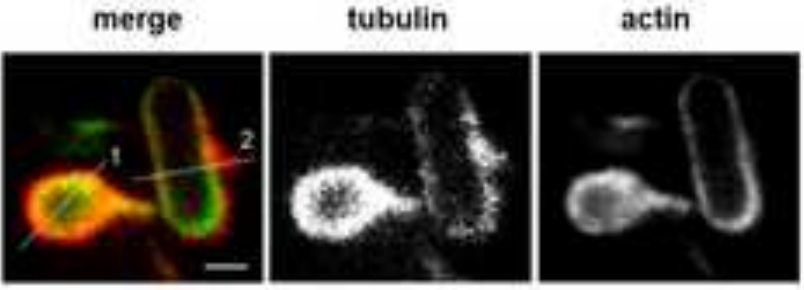

b

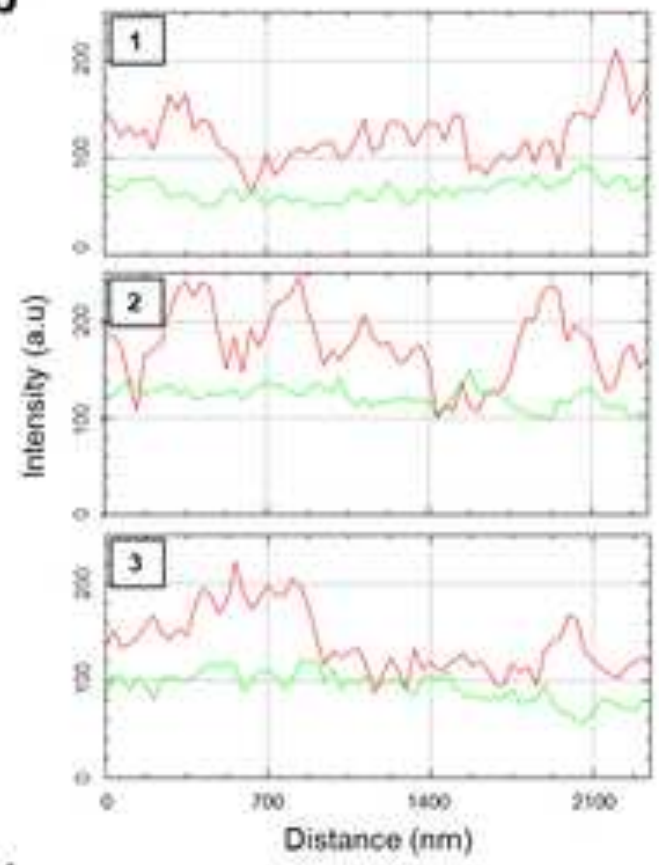

d
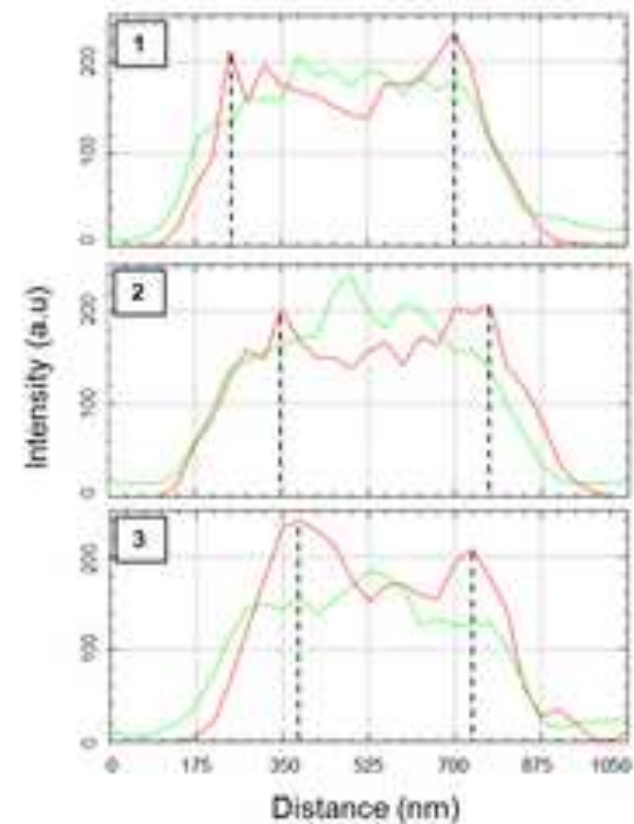

f
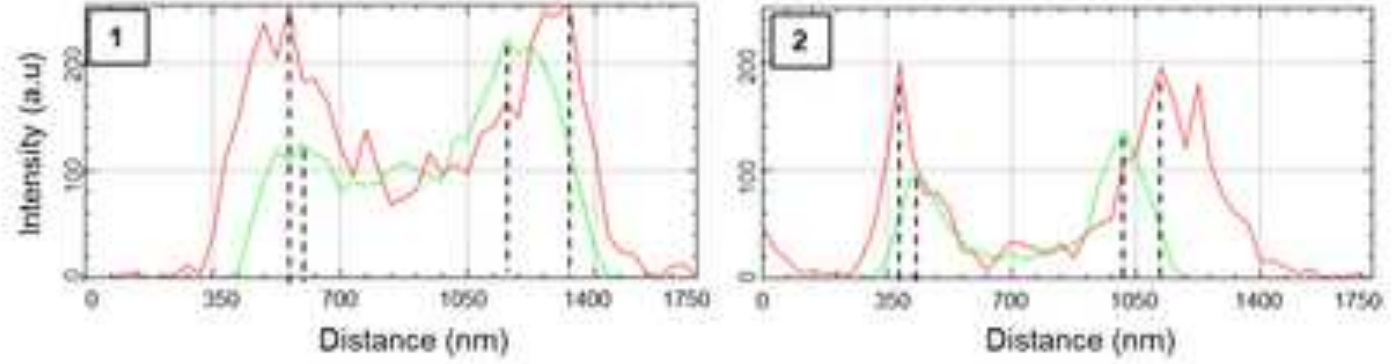
a

d

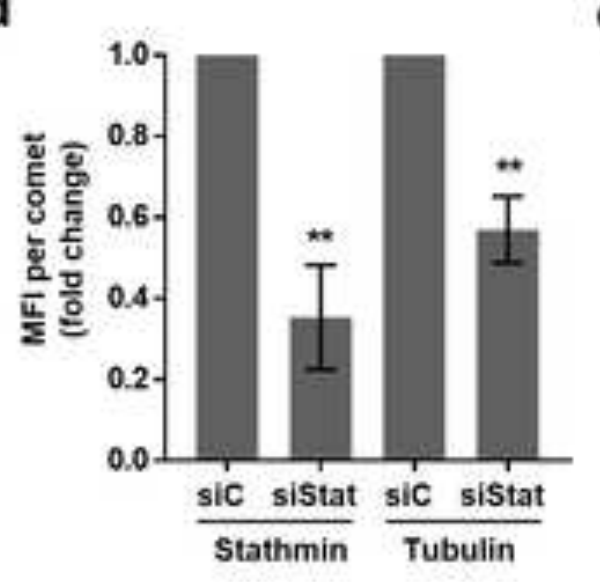

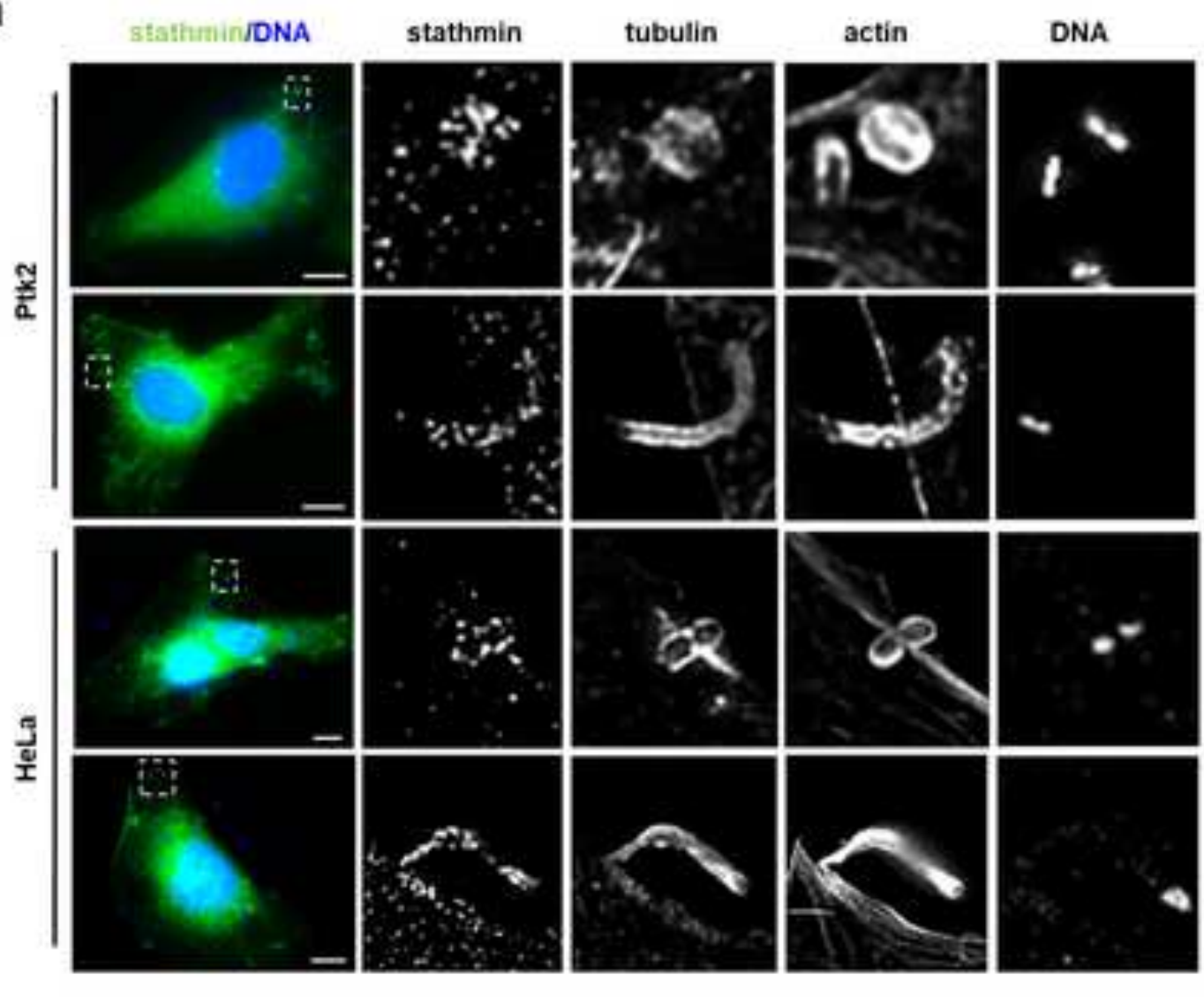

b

e

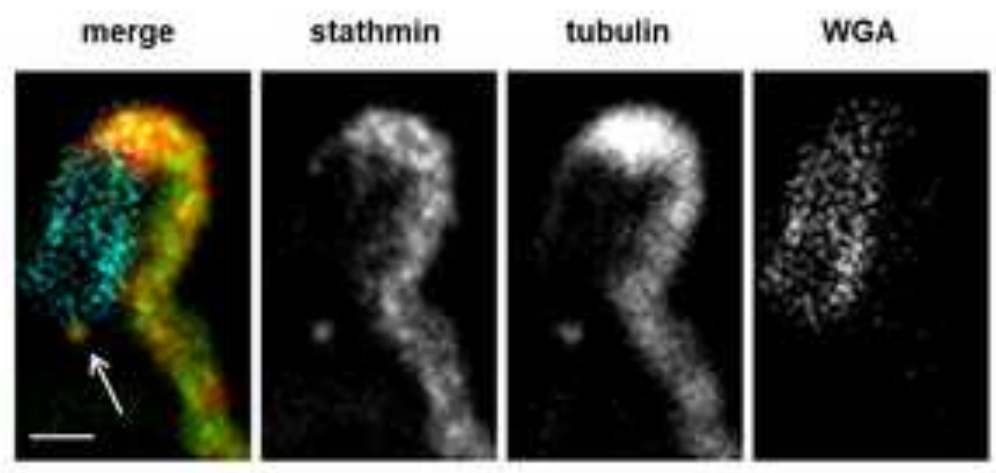

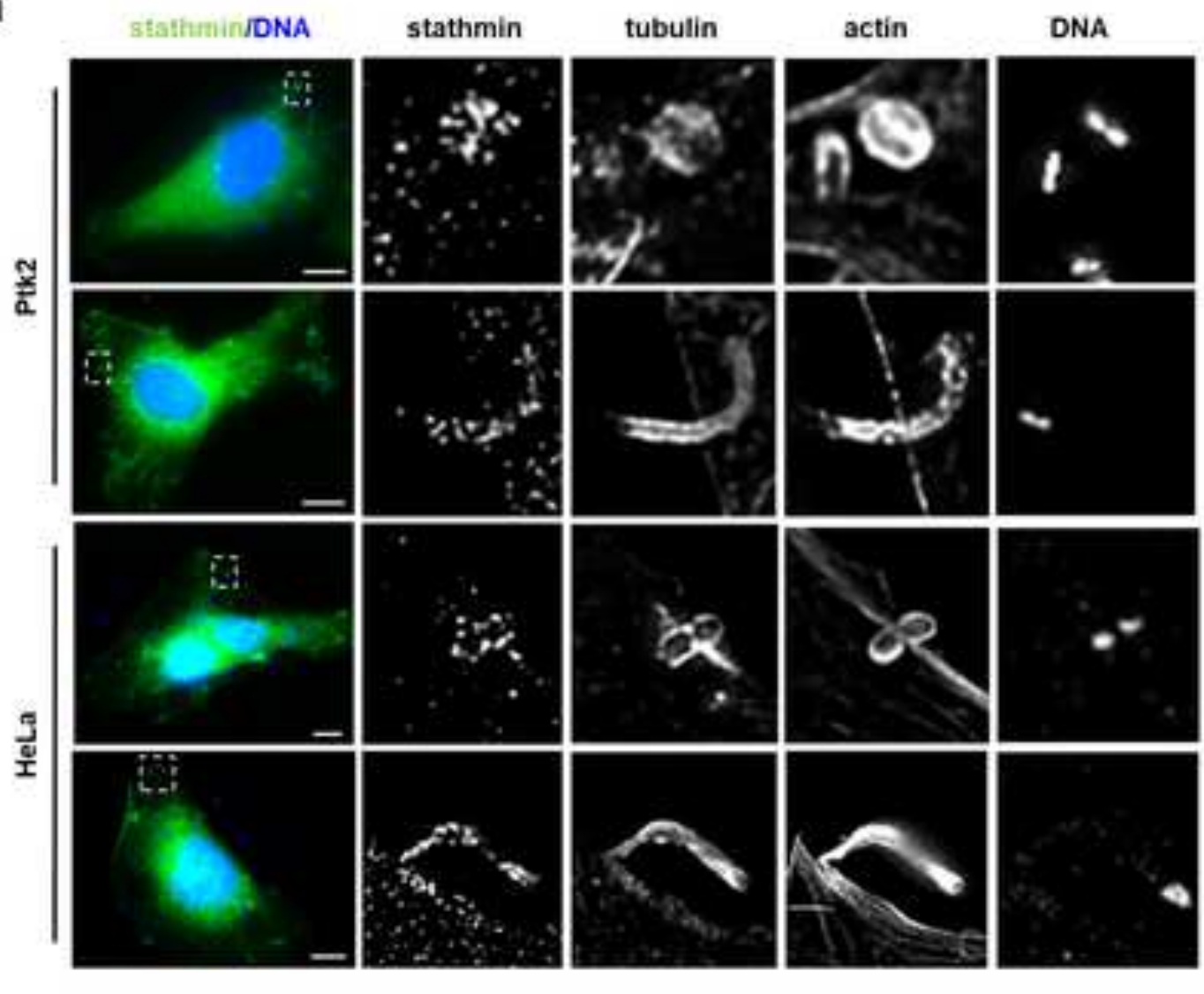

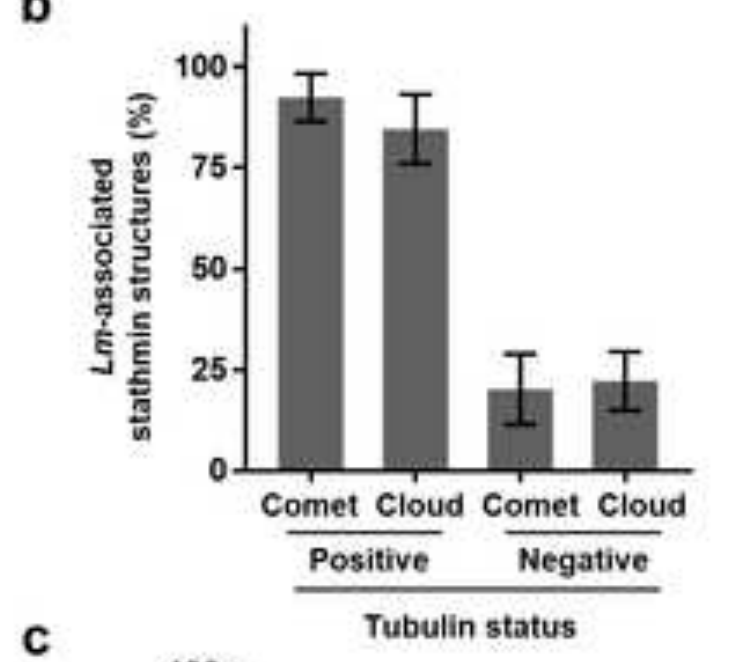

C

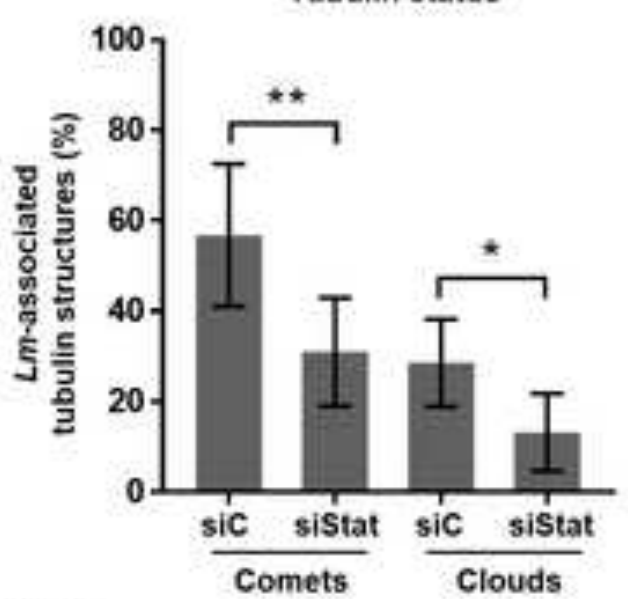


a

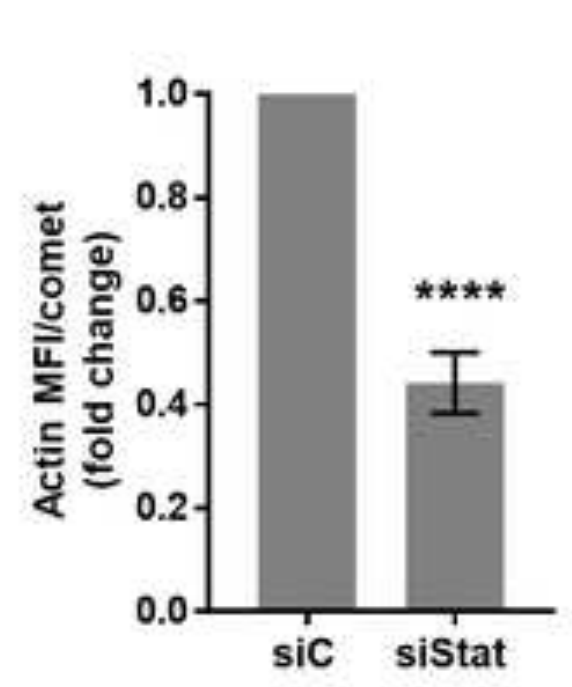

b

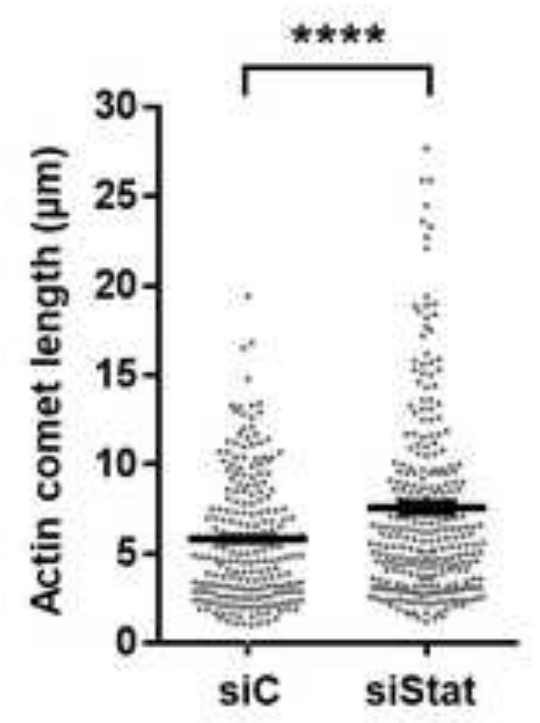

C

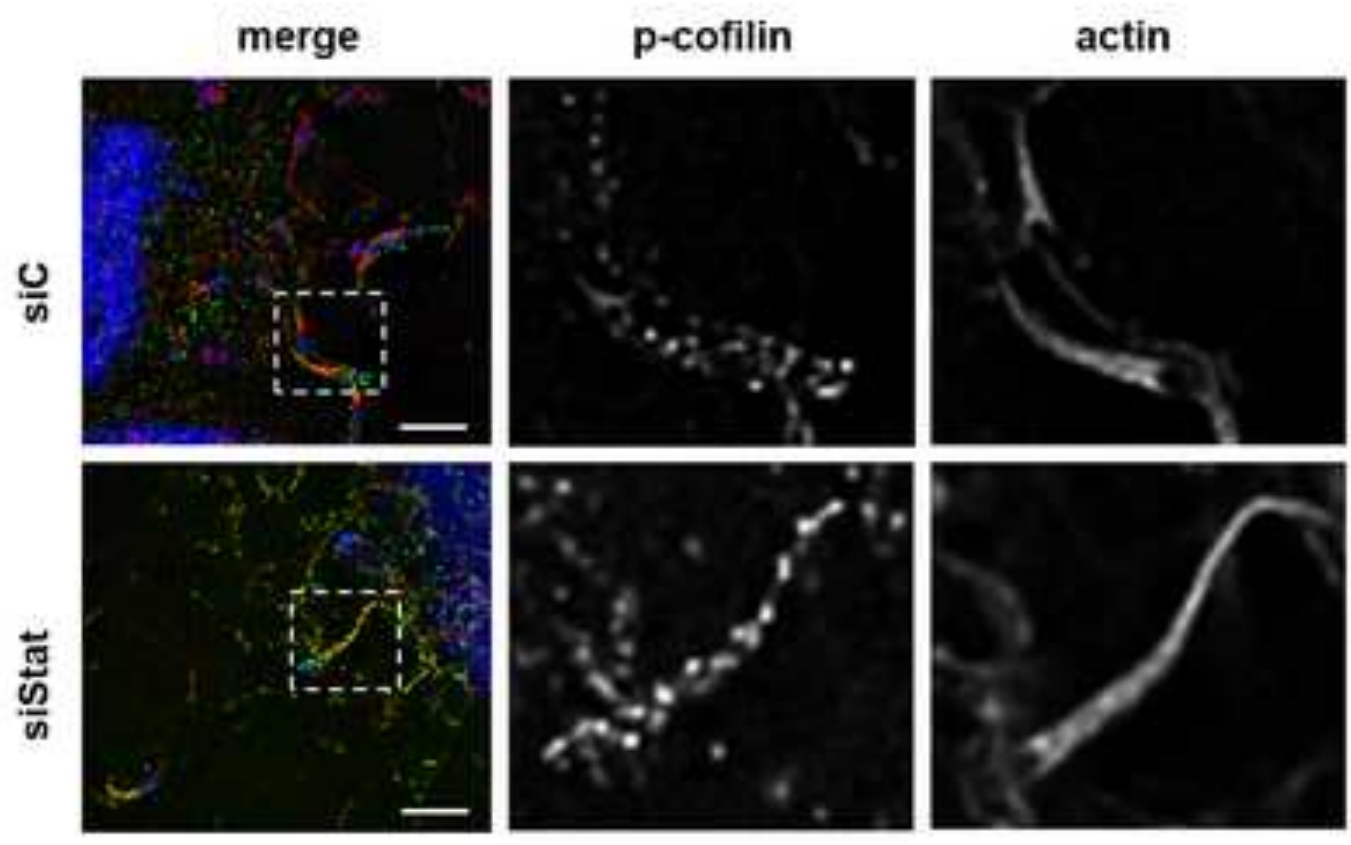

d

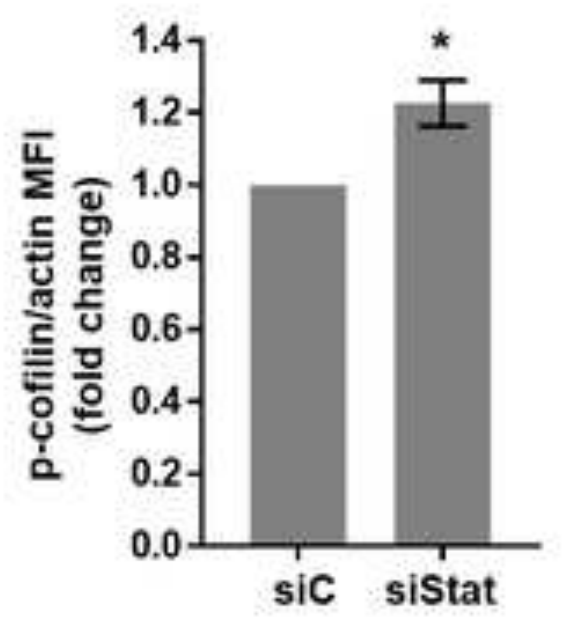


a
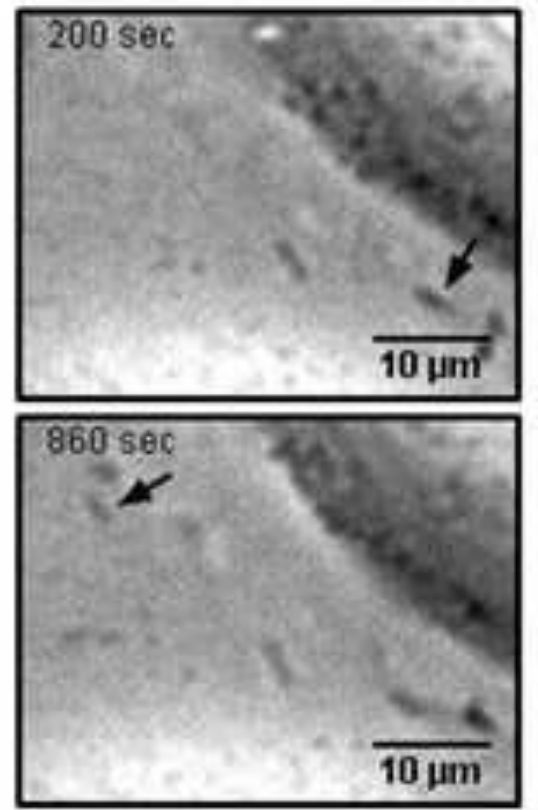

C

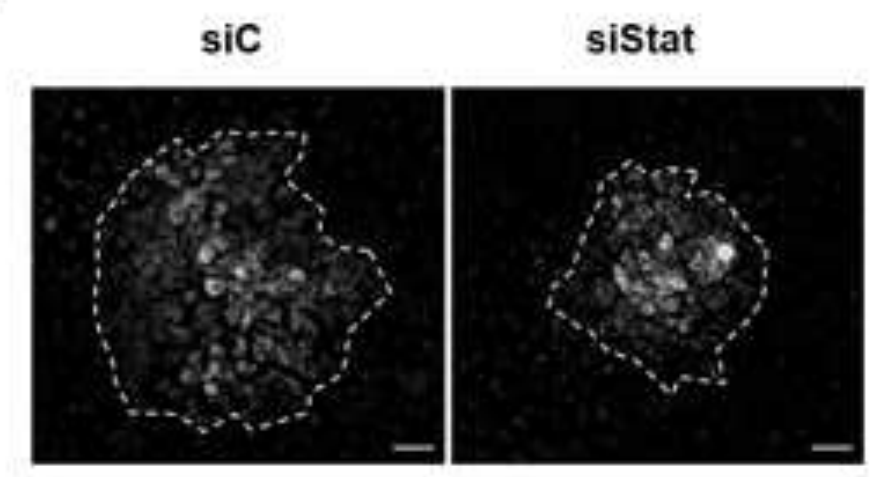

b

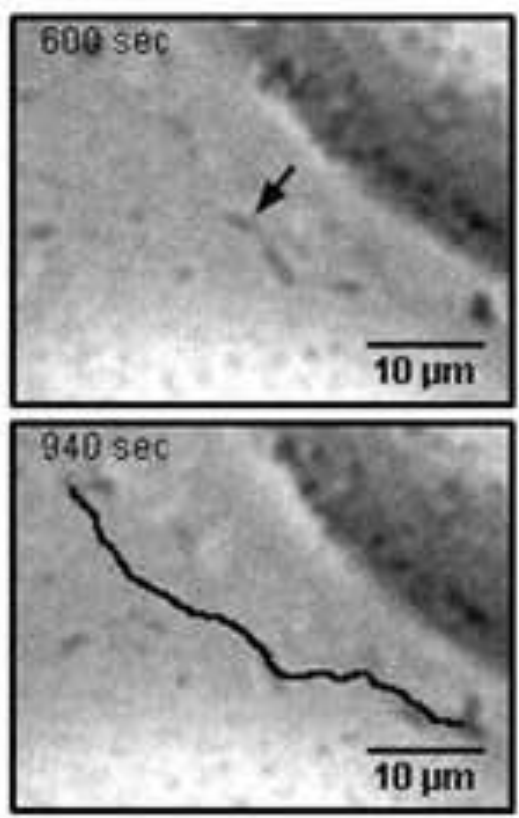

d

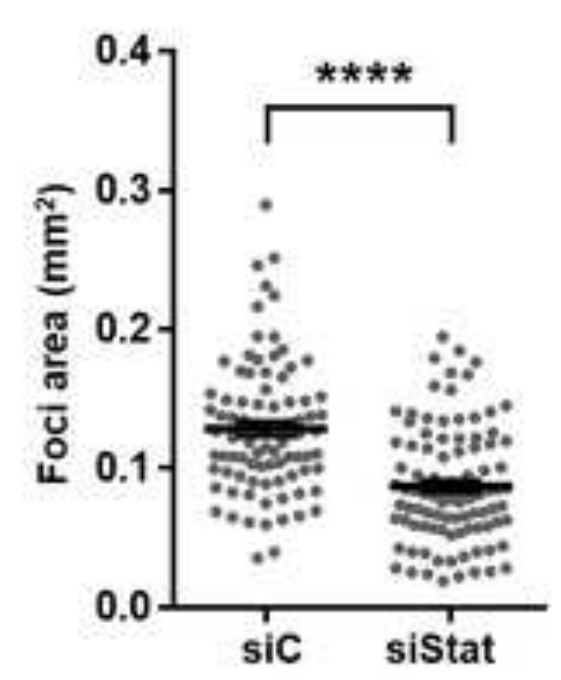

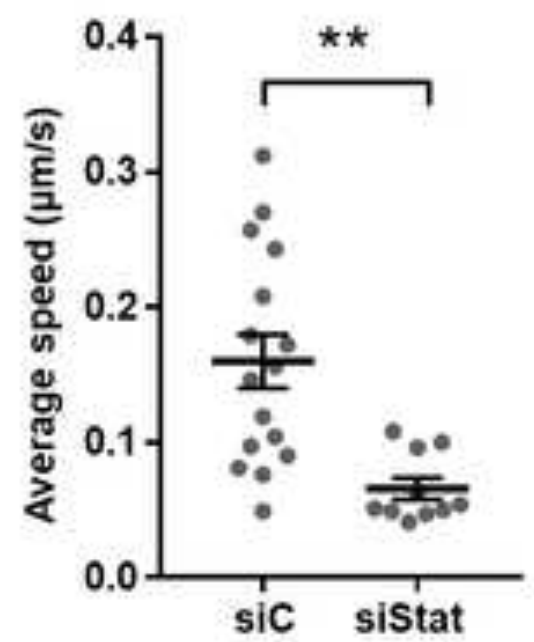

e

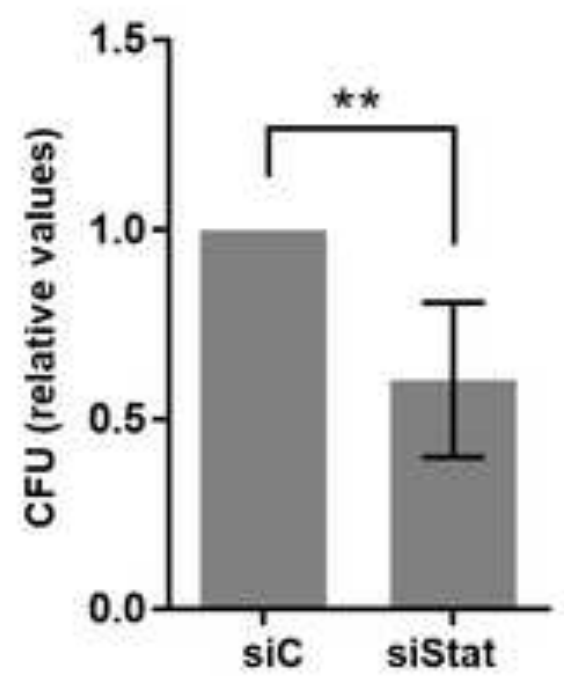




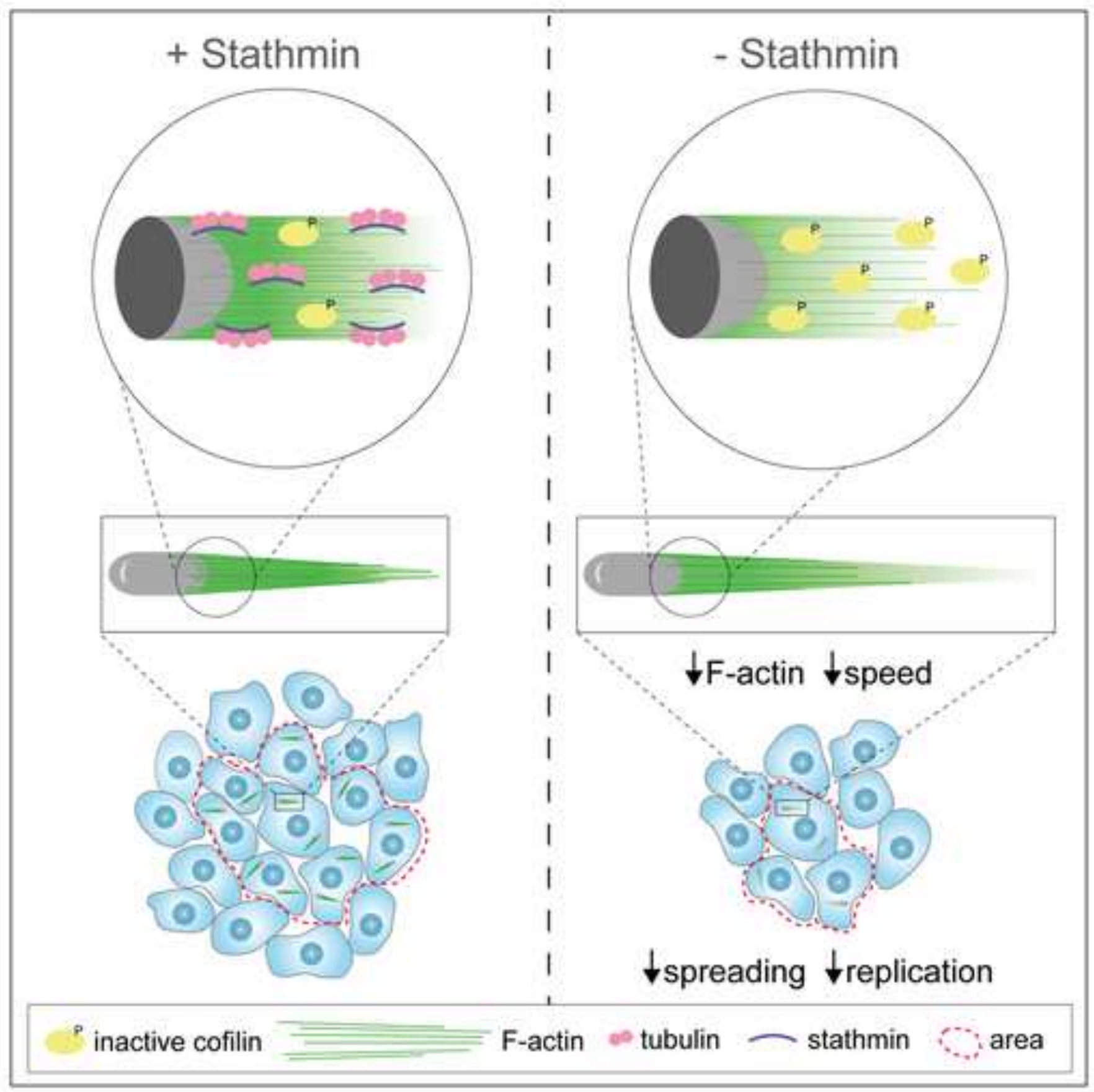




\begin{tabular}{ccccc} 
& \multicolumn{2}{c}{ Speed $(\mu \mathrm{m} / \mathrm{s})$} & & \\
\cline { 2 - 3 } Exp & siC & siStat & Fold change & p value \\
\hline$\# 1$ & $0.160 \pm 0.020$ & $0.066 \pm 0.008$ & 2.4 & 0.0014 \\
$\# 2$ & $0.051 \pm 0.031$ & $0.034 \pm 0.004$ & 1.6 & 0.0026 \\
$\# 3$ & $0.130 \pm 0.017$ & $0.082 \pm 0.012$ & 1.6 & 0.0198 \\
$\# 4$ & $0.065 \pm 0.012$ & $0.036 \pm 0.004$ & 1.8 & 0.0047
\end{tabular}


Foci area $\left(\mathrm{mm}^{2}\right)$

\begin{tabular}{ccccc} 
Exp & siC & siStat & Fold change & $p$ value \\
\hline$\# 1$ & $0.058 \pm 0.003$ & $0.049 \pm 0.002$ & 1.16 & 0.0300 \\
$\# 2$ & $0.166 \pm 0.005$ & $0.134 \pm 0.001$ & 1.24 & 0.0006 \\
$\# 3$ & $0.152 \pm 0.005$ & $0.129 \pm 0.005$ & 1.18 & 0.0001
\end{tabular}

